\title{
Boundary regularity of minimizers of $p(x)$-energy functionals
}

\author{
Maria Alessandra Ragusa ${ }^{a}$, Atsushi Tachikawa ${ }^{\mathrm{b}, *}$ \\ a Dipartimento di Matematica e Informatica, Università di Catania, Viale Andrea Doria, 6-95125 Catania, Italy \\ ${ }^{\mathrm{b}}$ Department of Mathematics, Faculty of Science and Technology, Tokyo University of Science, Noda, Chiba, 278-8510, Japan
}

Received 7 June 2014; received in revised form 3 November 2014; accepted 6 November 2014

Available online 13 November 2014

\section{Abstract}

The paper is devoted to the study of the regularity on the boundary $\partial \Omega$ of a bounded open set $\Omega \subset \mathbb{R}^{m}$ for minimizers $u$ for $p(x)$-energy functionals of the following type

$$
\mathcal{E}(u ; \Omega):=\int_{\Omega}\left(g^{\alpha \beta}(x) G_{i j}(u) D_{\alpha} u^{i}(x) D_{\beta} u^{j}(x)\right)^{p(x) / 2} d x
$$

where $\left(g^{\alpha \beta}(x)\right)$ and $\left(G_{i j}(u)\right)$ are symmetric positive definite matrices whose entries are continuous functions and $p(x) \geq 2$ is a continuous function. The authors prove that such minimizers $u$ have no singular points on the boundary.

๑ 2014 L'Association Publications de l'Institut Henri Poincaré. Published by Elsevier B.V. All rights reserved.

\section{Résumé}

Dans cet article, les auteurs étudient la régularité sur la frontière $\partial \Omega$ d'un ouvert borné $\Omega \subset \mathbb{R}^{m}$ des minimiseurs $u$ des fonctionnelles d'énergie $p(x)$ du type suivant :

$$
\mathcal{E}(u ; \Omega):=\int_{\Omega}\left(g^{\alpha \beta}(x) G_{i j}(u) D_{\alpha} u^{i}(x) D_{\beta} u^{j}(x)\right)^{p(x) / 2} d x,
$$

où $\left(g^{\alpha \beta}(x)\right)$ et $\left(G_{i j}(u)\right)$ sont des matrices symétriques définies positives dont les éléments sont des fonctions continues et $p(x) \geq 2$ est une fonction continue. Les auteurs prouvent que ces minimiseurs $u$ n'ont pas de point singulier sur la frontière $\partial \Omega$.

๑ 2014 L'Association Publications de l'Institut Henri Poincaré. Published by Elsevier B.V. All rights reserved.

MSC: 49N60; 35J50; 58E20

Keywords: $p(x)$-growth; Minimizer; Boundary regularity

\footnotetext{
* Corresponding author.

E-mail addresses: maragusa@dmi.unict.it (M.A. Ragusa), Tachikawa_atsushi@ma.noda.tus.ac.jp (A. Tachikawa).
} 


\section{Introduction}

Let $\Omega \subset \mathbb{R}^{m}(m \geq 2)$ be a bounded open set. For maps $u: \Omega \rightarrow \mathbb{R}^{n}$ we consider the $p(x)$-energy functional defined as

$$
\mathcal{E}(u ; \Omega):=\int_{\Omega}\left(g^{\alpha \beta}(x) G_{i j}(u) D_{\alpha} u^{i}(x) D_{\beta} u^{j}(x)\right)^{p(x) / 2} d x,
$$

where $\left(g^{\alpha \beta}(x)\right)$ and $\left(G_{i j}(u)\right)$ are symmetric positive definite matrices whose entries are continuous functions defined on $\Omega$ and $\mathbb{R}^{n}$ respectively, and $p(x)$ in a continuous function on $\Omega$ with $p(x) \geq 2$. Greek indices $\alpha, \beta, \ldots$ are to be summed from 1 to $m$, and Latin indices $i, j, \ldots$ from 1 to $n$. The Einstein summation convention is used. In the following we write, for the integrand of (1.1),

$$
e(u)(x):=g^{\alpha \beta}(x) G_{i j}(u) D_{\alpha} u^{i}(x) D_{\beta} u^{j}(x) .
$$

The aim of this paper is to study the boundary regularity of the minimizers of the $p(x)$-energy functionals.

The functional $\mathcal{E}$ is a particular case of the functionals of the type

$$
\mathcal{F}(u ; \Omega)=\int_{\Omega} f(x, u, D u) d x,
$$

where $f: \Omega \times \mathbb{R}^{n} \times \mathbb{R}^{m n} \rightarrow \mathbb{R}$ is a Carathéodory function satisfying the following so-called $(p, q)$-growth condition: there exist constants $\Lambda \geq \lambda>0, q \geq p \geq 1$ such that

$$
\lambda|\xi|^{p} \leq f(x, u, \xi) \leq \Lambda\left(1+|\xi|^{q}\right)
$$

for all $(x, u, \xi) \in \Omega \times \mathbb{R}^{n} \times \mathbb{R}^{m n}$. We call $\mathcal{F}$ a functional with standard growth if $p=q$, and with non-standard growth if $q>p$. If the integrand $f=f_{p(x)}$ satisfies

$$
\lambda|\xi|^{p(x)} \leq f_{p(x)}(x, u, \xi) \leq \Lambda\left(1+|\xi|^{p(x)}\right),
$$

for all $(x, u, \xi) \in \Omega \times R^{n} \times \mathbb{R}^{m n}$, then

$$
\mathcal{F}_{p(x)}(u ; \Omega):=\int_{\Omega} f_{p(x)}(x, u, D u) d x
$$

is called a functional with $p(x)$-growth. The $p(x)$-energy functional $\mathcal{E}$ is a $p(x)$-growth functional with a special structure.

Non-standard growth problems are attracting great interest, since Marcellini treated them in [13]. Especially, in the last two decades, about the regularity of minimizers for $p(x)$-growth functionals, considerable progress has been made. In 1995, Zhikov [17] studied Lavrentiev phenomenon for the functional

$$
\mathcal{D}_{p(x)}(u):=\int_{\Omega}|D u|^{p(x)} d x .
$$

He also obtained higher integrability results for the minimizers of $\mathcal{D}_{p(x)}$ in [18]. On the regularity of minimizers of $\mathcal{D}_{p(x)}$, a fundamental result was established by Coscia and Mingione [4] in 1999. They proved that a minimizer $u$ of $\mathcal{D}_{p(x)}$ is in the class $C^{1, \alpha}(\Omega)$ under the condition that $p(x)$ is Hölder continuous.

For general $p(x)$-growth functionals, interior partial regularity results are obtained in [1-3,7-9].

For $p(x)$-energy $\mathcal{E}$, Ragusa, Tachikawa and Takabayashi [15] obtained interior partial regularity of minimizers; they showed that the singular set $S_{u}$ of a minimizer $u$ can have Hausdorff dimension $\operatorname{dim}^{\mathcal{H}}\left(S_{u}\right)$ at most $m$-inf $p(x)$. In [14] the interior everywhere regularity was shown under the so-called one-sided condition. In [16], assuming the boundedness of a minimizer $u$, the second author improved the estimate on the Hausdorff dimension of $S_{u}$ as $\operatorname{dim}_{\mathcal{H}}\left(S_{u}\right) \leq m$-[inf $\left.p(x)\right]-1$, where [] stands for the Gauss symbol.

In this paper, we treat boundary regularity of minimizers for $p(x)$-energy $\mathcal{E}$. For standard growth case, Jost and Meier [12] proved that a minimizers for certain quadratic functionals cannot have singular points on the boundary. 
Duzaar, Grotowski and Kronz [6] generalized this result to general $p$-energy functionals

$$
\int_{\Omega}\left(g^{\alpha \beta}(x) G_{i j}(x, u) D_{\alpha} u^{i}(x) D_{\beta} u^{j}(x)\right)^{p / 2} d x,
$$

for $p>1$. The aim of this paper is to obtain such kind of boundary regularity results for $p(x)$-energy.

Now, let us introduce some conditions and definitions in order to state the main result. We consider the following conditions on $g^{\alpha \beta}(x), G_{i j}(u)$ and $p(x)$.

(C1) There exist positive constants $\lambda_{g}, \Lambda_{g}, \lambda_{G}, \Lambda_{G}$ such that

$$
\lambda_{g}|\zeta|^{2} \leq g^{\alpha \beta}(x) \zeta_{\alpha} \zeta_{\beta} \leq \Lambda_{g}|\zeta|^{2}, \quad \lambda_{G}|\eta|^{2} \leq G_{i j}(u) \eta^{i} \eta^{j} \leq \Lambda_{G}|\eta|^{2}
$$

for all $x \in \Omega, \zeta \in \mathbb{R}^{m}$ and $u, \eta \in \mathbb{R}^{n}$.

(C2) The exponent $p(x)$ and the coefficients $g^{\alpha \beta}(x), G_{i j}(u)$ are Hölder continuous; there exist positive constants $\tau, \tau^{\prime}, \sigma<1, L_{p}, L_{g}$, and $L_{h}$ such that

$$
\begin{aligned}
& |p(x)-p(y)| \leq L_{p}|x-y|^{\sigma} / 2=: \omega_{p}(|x-y| / 2) \quad \text { for all } x, y \in \Omega, \\
& \left|g^{\alpha \beta}(x)-g^{\alpha \beta}(y)\right| \leq L_{g}|x-y|^{\tau}=: \omega_{g}(|x-y|) \quad \text { for all } x, y \in \Omega, \\
& \left|G_{i j}(u)-G_{i j}(v)\right| \leq L_{h}|u-v|^{\tau^{\prime}}=: \omega_{G}\left(|u-v|^{2}\right) \quad \text { for all } u, v \in \mathbb{R}^{n} .
\end{aligned}
$$

(C3) The exponent $p(x)$ satisfies

$$
2 \leq \gamma_{1}:=\inf _{x \in \Omega} p(x) \leq \sup _{x \in \Omega} p(x)=: \gamma_{2}<+\infty .
$$

Let $\Omega \subset \mathbb{R}^{m}$ be a bounded domain with smooth boundary $\partial \Omega$. In the following, for a function $w: \Omega \rightarrow \mathbb{R}^{k}$ and a measurable set $D \subset \Omega$, we write

$$
\int_{D} w(x) d x:=\frac{1}{|D|} \int_{D} w(x) d x
$$

where $|D|$ denotes the Lebesgue measure of $D$. For a ball $B(y, r):=\left\{x \in \mathbb{R}^{m} ;|x-y|<0\right\}$, we write

$$
w_{y, r}:=f_{B(y, r) \cap \Omega} w(x) d x .
$$

When there is no doubt of confusion, we omit the center $y$ and set $w_{r}:=w_{y, r}$.

Let us define some function spaces. For a bounded open set $\Omega \subset \mathbb{R}^{m}$ and a function $p: \Omega \rightarrow[1,+\infty)$, we define $L^{p(x)}(\Omega)$ and $W^{1, p(x)}(\Omega)$ as follows:

$$
\begin{aligned}
& L^{p(x)}:=\left\{u \in L^{1}(\Omega) ; \int_{\Omega}|u|^{p(x)} d x<+\infty\right\} . \\
& W^{1, p(x)}:=\left\{u \in L^{p(x)} \cap W^{1,1}(\Omega) ; D u \in L^{p(x)}(\Omega)\right\} .
\end{aligned}
$$

We also define $L_{\mathrm{loc}}^{p(x)}(\Omega)$ and $W_{\mathrm{loc}}^{1, p(x)}(\Omega)$ similarly.

As mentioned in [5], if $p(x)$ is uniformly continuous and $\partial \Omega$ satisfies uniform cone property, then

$$
W^{1, p(x)}(\Omega)=\left\{u \in W^{1,1}(\Omega) ; D u \in L^{p(x)}(\Omega)\right\} .
$$

In any case, if $p(x)$ is continuous in $\Omega$, we have

$$
W_{\mathrm{loc}}^{1, p(x)}(\Omega)=\left\{u \in W_{\mathrm{loc}}^{1,1}(\Omega) ;|D u|^{p(x)} \in L_{\mathrm{loc}}^{1}(\Omega)\right\} .
$$

We also define

$$
W_{0}^{1, p(x)}(\Omega):=\left\{u \in W_{0}^{1,1}(\Omega) ; \int_{\Omega}|D u|^{p(x)} d x<\infty\right\},
$$


and for a given map $\varphi$

$$
\varphi+W_{0}^{1, p(x)}(\Omega):=\left\{u \in W^{1, p(x)}(\Omega) ; u-\varphi \in W_{0}^{1, p(x)}(\Omega)\right\} .
$$

A map $u \in W_{\text {loc }}^{1, p(x)}(\Omega)$ is called to be a local minimizer of $\mathcal{F}_{p(x)}$ if it satisfies

$$
\mathcal{F}_{p(x)}(u ; \operatorname{supp} \varphi) \leq \mathcal{F}_{p(x)}(u+\varphi ; \operatorname{supp} \varphi),
$$

for any $\varphi \in W_{0}^{1, p(x)}(\Omega)$ with compact support in $\Omega$.

Theorem 1.1. Let $\Omega \subset \mathbb{R}^{m}$ be a bounded domain with Lipschitz boundary $\partial \Omega$. Assume that $g^{\alpha \beta}(x), G_{i j}(u)$ and $p(x)$ satisfy the conditions $(C 1)-(C 3)$ on $\Omega$. Let $u \in W^{1, p(x)}(\Omega)$ be a bounded minimizer of the functional $\mathcal{E}(v ; \Omega)$ defined by

$$
\mathcal{E}(v ; \Omega):=\int_{\Omega} g^{\alpha \beta}(x) G_{i j}(u) D_{\alpha} u^{i}(x) D_{\beta} u^{j}(x) d x,
$$

in the class

$$
h+W_{0}^{1, p(x)}(\Omega):=\left\{v \in W^{1, p(x)}(\Omega) ; v-h \in W_{0}^{1, p(x)}(\Omega)\right\},
$$

for a given boundary data $h \in W^{1, s}(\Omega)$ for some $s>m$. Then $u$ is Hölder continuous near the boundary $\partial \Omega$.

\section{Notation and preliminary results}

Throughout this paper we use the following notation: for $x_{0}=\left(x_{0}^{1}, \ldots, x_{0}^{m-1}, 0\right)$ and $r>0$, we put

$$
\begin{aligned}
& B^{+}\left(x_{0}, r\right):=\left\{x \in \mathbb{R}^{m} ;\left|x-x_{0}\right|<r, x^{m}>0\right\}, \\
& \Gamma\left(x_{0}, r\right):=\left\{x \in \mathbb{R}^{m} ;\left|x-x_{0}\right|<r, x^{m}=0\right\}, \\
& \partial^{+} B^{+}\left(x_{0}, r\right):=\partial B^{+}\left(x_{0}, r\right) \backslash \Gamma\left(x_{0}, r\right) .
\end{aligned}
$$

When $x_{0}=0$, we omit the center $x_{0}=0$ and write simply

$$
B^{+}(r):=B^{+}(0, r), \quad \Gamma(r):=\Gamma(0, r), \quad \partial^{+} B^{+}(r):=\partial^{+} B^{+}(0, r) .
$$

For $x \in B^{+}\left(x_{0}, R\right)$ and $r<\operatorname{dist}\left(x, \partial B\left(x_{0}, R\right)\right)=R-\left|x-x_{0}\right|$, we put

$$
\begin{aligned}
& \Omega(x, r):=B(x, r) \cap B^{+}\left(x_{0}, R\right), \\
& p_{1}(x, r):=\inf _{\Omega(x, r)} p(y), \quad p_{2}(x, r):=\sup _{\Omega(x, r)} p(y) .
\end{aligned}
$$

For $p_{1}$ and $p_{2}$, when the center $x=x_{0}$ is clearly understood, we abbreviate as

$$
p_{1}(r):=p_{1}(x, r), \quad p_{2}(r):=p_{2}(x, r) .
$$

When we consider the behavior of the solution near the boundary point $x_{0} \in \partial \Omega$, we flatten the $\partial \Omega$ so that $x_{0}=$ $(0, \ldots, 0), B\left(x_{0}, R_{1}\right) \cap \Omega=B^{+}\left(0, R_{1}\right)$ for some $R_{1}>0$ and $\partial \Omega \cap B\left(x_{0}, R_{1}\right)=\Gamma\left(0, R_{1}\right)$.

We use $c$ without subscript as generic constants, which may change from line to line, but does not depend on the crucial quantities.

Let $\omega_{1}:[0,+\infty) \rightarrow[0,+\infty)$ be a nondecreasing continuous function with $\omega_{1}(0)=0$ which represents the modulus of continuity, namely $\omega_{1}$ satisfies

$$
|p(x)-p(y)| \leq \omega_{1}(|x-y|) .
$$

Let us consider the following condition on $\omega_{1}$.

$$
\lim _{r \rightarrow 0} \omega_{1}(r) \log \left(\frac{1}{r}\right)=\mu_{0}<+\infty .
$$


The above condition implies

$$
(1 / t)^{\omega_{1}(t)}=\exp \left(-\log t \omega_{1}(t)\right) \rightarrow e^{\mu_{0}} \quad \text { as } t \rightarrow 0^{+} .
$$

When $\omega_{1}$ satisfies (2.3), we say that $p(x)$ is logarithmic continuous. We mention also that if $p(x)$ is Hölder continuous, then the condition (2.3) is fulfilled.

For a continuous function $p(x)>1$ on $\Omega$ satisfying (2.2) with (2.3), let $f_{p(x)}(x, u, \xi)$ be a Carathéodory function on $\Omega \times \mathbb{R}^{n} \times \mathbb{R}^{m n}$ which satisfies the growth condition (1.5). We define $\mathcal{F}_{p(x)}(w, \Omega)$ by (1.6).

Let us begin with remembering the following higher integrability results on local minimizers that is originally shown by Zhikov [18] and is generalized by Acerbi and Mingione [1].

Proposition 2.1. (See [7, Theorem 3.1].) Let $\mathcal{F}_{p(x)}$ be a functional as above. Assume that the exponent $p(x)>1$ has modulus of continuity $\omega_{1}$ which satisfies (2.3). Let $u \in W_{\text {loc }}^{1, p(x)}\left(\Omega, \mathbb{R}^{n}\right)$ be a local minimizer of $\mathcal{F}_{p(x)}$. Then, there exists a constant $\delta>0$ such that $|D u|^{(1+\delta) p(x)} \in L_{\mathrm{loc}}^{1}(\Omega)$. Moreover, the estimate

$$
f_{B(y, R)}|D u|^{(1+\delta) p(x)} d x \leq c_{0}\left(f_{B(y, 2 R)}\left(1+|D u|^{2}\right)^{p(x) / 2} d x\right)^{(1+\delta)}
$$

holds for any $B(y, 2 R) \Subset \Omega$.

When we consider the functional $\mathcal{F}_{p(x)}$ on $B^{+}(T)$, as in [12] for the case $p(x)=2$, let us call a map $v \in$ $\bigcap_{T^{\prime}<T} W^{1, p(x)}\left(B^{+}\left(T^{\prime}\right), \mathbb{R}^{n}\right)$ a local minimizer of the functional

$$
\mathcal{F}_{p(x)}\left(w, B^{+}(T)\right)=\int_{B^{+}(T)} f_{p(x)}(x, w, D w) d x
$$

in $B^{+}(T) \cup \Gamma(T)$, if for every $T^{\prime}<T$ and any $\varphi \in W_{0}^{1,1}\left(B^{+}\left(T^{\prime}\right), \mathbb{R}^{n}\right)$ the following inequality holds:

$$
\mathcal{F}_{p(x)}\left(v, B^{+}\left(T^{\prime}\right)\right) \leq \mathcal{F}_{p(x)}\left(v+\varphi, B^{+}\left(T^{\prime}\right)\right) .
$$

Then we have the following lemma on the higher integrability of such local minimizers.

Proposition 2.2. (See [15, Lemma 3.2].) Assume that the exponent $p(x)>1$ has modulus of continuity $\omega_{1}$ which satisfies (2.3). Let $p_{1}:=\inf _{B^{+}(T)} p(x)$ and $p_{2}:=\sup _{B^{+}(T)} p(x)$, and suppose that

$$
\left(p_{2}\right)_{*}=\frac{m p_{2}}{m+p_{2}}<p_{1} \quad\left(\text { or equivalently } p_{1}^{*}=\frac{m p_{1}}{m-p_{1}}>p_{2}\right) .
$$

For some $\varepsilon>0$, let $h$ be a given map in the class $W^{1,(1+\varepsilon) p(x)}\left(B^{+}(T)\right)$. Let $v$ be a local minimizer of $\mathcal{F}_{p(x)}$ in the class

$$
\left\{w \in W^{1, p(x)}\left(B^{+}(T), \mathbb{R}^{n}\right) ; w=h \text { on } \Gamma(T)\right\} .
$$

Then, there exists a positive constant $\hat{\delta}<\varepsilon$ such that for any $\delta \in(0, \hat{\delta})$ the local minimizer $v$ satisfies $v \in$ $W^{1,(1+\delta) p(x)}\left(B^{+}\left(T^{\prime}\right)\right)$ for any $T^{\prime}<T$. Moreover, if $x_{0} \in B^{+}\left(T^{\prime}\right) \cup \Gamma\left(T^{\prime}\right)$ and $r<T-T^{\prime}$, we have

$$
\begin{aligned}
& \left(f_{\Omega\left(x_{0}, r / 2\right)}\left(1+|D v|^{2}\right)^{(1+\delta) p(x) / 2} d x\right)^{1 /(1+\delta)} \\
& \leq c_{1} f_{\Omega\left(x_{0}, r\right)}\left(1+|D v|^{2}\right)^{p(x) / 2} d x+c_{2}\left(f_{\Omega\left(x_{0}, r\right)}\left(1+|D h|^{2}\right)^{(1+\delta) p(x) / 2} d x\right)^{1 /(1+\delta)},
\end{aligned}
$$

where we put $\Omega(y, \rho):=B(y, \rho) \cap B^{+}(T)$.

By virtue of Propositions 2.1 and 2.2, we have the following estimate of the minimizer. 
Corollary 2.3. (See [15, Corollary 3.3].) Let $D \subset \mathbb{R}^{m}$ be a bounded domain with smooth boundary $\partial$ D. Let $S>0$ be a positive number which satisfies the following conditions.

(S1) $p_{1}=p_{1}(x, 4 S)$ and $p_{2}=p_{2}(x, 4 S)$ satisfy $(2.6)$.

(S2) There is a diffeomorphism $\psi: B(y, 4 S) \rightarrow B(T)$ which satisfies

$$
\psi(B(y, 4 S) \cap D) \subset B^{+}(T) \quad \text { and } \quad \psi(B(y, 4 S) \cap \partial D)=\Gamma(T) .
$$

Assume that $p(x), h(x)$ satisfy assumptions in Proposition 2.2, $v$ be a minimizer of $\mathcal{F}_{p(x)}(\cdot, D)$ in the class

$$
\left\{w \in W^{1, p(x)}\left(D, \mathbb{R}^{n}\right) ; w-h \in W_{0}^{1,1}\left(D ; \mathbb{R}^{n}\right)\right\} .
$$

Then there exists a constant $\hat{\delta} \in(0, \varepsilon)$ such that for any $\delta \in(0, \hat{\delta}]$, we have that $v \in W^{p(x)(1+\delta)}\left(D, \mathbb{R}^{n}\right)$ and that

$$
\int_{D}\left(1+|D v|^{2}\right)^{(1+\delta) p(x) / 2} d x \leq c_{3}\left(1+|D|^{\delta} S^{-m \delta}\right) \int_{D}\left(1+|D h|^{2}\right)^{(1+\delta) p(x) / 2} d x,
$$

where $c_{3}$ depends only on $m, \lambda, \Lambda, p(x), \mathcal{F}_{p(x)}(h, D)$.

We also mention that we have Caccioppoli-type inequality with boundary value by [15, (3.14)].

Lemma 2.4. Let $v$ be a minimizer of $\mathcal{F}_{p(x)}(\cdot, \Omega)$ in the class

$$
\left\{w \in W^{1, p(x)}\left(\Omega, \mathbb{R}^{n}\right) ; w=h \text { on } \partial \Omega\right\} .
$$

Then we have

$$
\int_{\Omega_{r / 2}}|D v|^{p(x)} d x \leq c_{4}\left(\int_{\Omega_{r}}\left(\frac{|v-h|}{r}\right)^{p(x)} d x+\int_{\Omega_{r}}|D h|^{p(x)} d x\right),
$$

where $c_{4}$ depends only on $\lambda, \Lambda$ and $p(x)$.

Using the above lemma and Corollary 2.3 with $D=\Omega$, it comes out the following estimates for the derivatives of bounded minimizers.

Corollary 2.5. Let $v$ be as in the previous lemma. Assume that the boundary condition $h$ satisfies

$$
\int_{\Omega(y, r)}|D h|^{(1+\varepsilon) p(x)} d x \leq c_{h} r^{-\gamma_{1}}
$$

for some constants $\varepsilon \in(0,1)$ and $c_{h}>0$, and that for some positive constant $M$

$$
\underset{x \in \Omega}{\operatorname{esssup}}|v(x)|, \quad \underset{x \in \Omega}{\operatorname{esssup}}|h(x)| \leq M
$$

hold for some positive constant $M$. Then, we have the following estimates for some constants $c_{5}$ and $c_{6}$ depending only on $\lambda, \Lambda, p(x)$, and $c_{h}$.

$$
\begin{aligned}
& f_{\Omega(y, r)}|D v|^{p(x)} d x \leq c_{5} r^{-p_{2}(y, 2 r),} \\
& f_{\Omega(y, r)}|D v|^{(1+\delta) p(x)} d x \leq c_{6} r^{-p_{2}(y, 4 r)(1+\delta)},
\end{aligned}
$$

where $\delta$ is arbitrary constant with $\delta \in(0, \hat{\delta})$ for $\hat{\delta}$ in Corollary 2.3 .

Proof. Without loss in generality we can assume that $c_{h}, M \geq 1$ and that $r \in(0,1)$. Since $p_{2}(2 r)=p_{2}(y, 2 r) \geq$ $p(x) \geq \gamma_{1}$ in $\Omega(y, 2 r)$, from (2.9) and the assumptions on $v$ and $h$, we have 


$$
\begin{aligned}
f_{\Omega(y, r)}|D v|^{p(x)} d x & \leq c\left(f_{\Omega(y, 2 r)}\left|\frac{v(x)-h(x)}{2 r}\right|^{p(x)} d x+c_{h}(2 r)^{-\gamma_{1}}\right) \\
& \leq c\left(M^{p_{2}(2 r)} r^{-p_{2}(2 r)}+c_{h}(2 r)^{-p_{2}(2 r)}\right) \\
& \leq c_{5} r^{-p_{2}(2 r)},
\end{aligned}
$$

for some positive constant $c_{5}$ depending only on $M$ and $c_{h}$. This is nothing but (2.11).

From (2.7), (2.10) and (2.11), we have

$$
\begin{aligned}
f_{\Omega(y, 2 r)}|D v|^{(1+\delta) p(x)} d x \leq & c_{1}\left(f_{\Omega(y, 2 r)}\left(1+|D v|^{2}\right)^{p(x) / 2} d x\right)^{(1+\delta)} \\
& +c_{2}\left(f_{\Omega(y, r)}\left(1+|D h|^{2}\right)^{(1+\delta) p(x) / 2} d x\right)^{1 /(1+\delta)} \\
\leq & c_{6} r^{-p_{2}(4 r)(1+\delta)} .
\end{aligned}
$$

Thus we get (2.12) also.

In what follows, we are fixing a constant $\delta \in(0,1)$ so that the above lemmas and propositions hold and that

$$
m \delta<\sigma
$$

We prepare the boundary version of the regularity result by Coscia and Mingione [4] for minimizers of the functional

$$
\mathcal{D}_{p(x)}(w, D):=\int_{D}|D w|^{p(x)} d x .
$$

Theorem 2.6. Assume that $p(x)$ satisfies (1.9). Let $R>0$ be sufficiently small so that

$$
\left(1+\frac{\delta}{2}\right) p_{2}(2 R) \leq(1+\delta) p_{1}(2 R) .
$$

Let $v \in W^{1, p(x)}\left(B^{+}(R), \mathbb{R}^{n}\right)$ a local minimizer of $\mathcal{D}_{p(x)}$ in the class

$$
\left\{w \in W^{1, p(x)} ; w=h \text { on } \Gamma(R)\right\},
$$

where $h$ is a given boundary data in the class $W^{1, s}\left(B^{+}(R), \mathbb{R}^{n}\right) s>(1+\delta) p_{2}$. Assume that $\mathcal{D}_{p(x)}(v) \leq K$ for some positive constant $K$. Then, for any $\varepsilon \in\left(0, m p_{2}(2 R) / s\right)$, we have

$$
\begin{aligned}
\int_{B^{+}(\rho)}|D v|^{p_{2}(2 R)} d x \leq & c_{7}\left[\left(\frac{\rho}{R}\right)^{m-\varepsilon} \int_{B^{+}(R)}|D v|^{p_{2}(2 R)} d x\right. \\
& \left.+\rho^{m-m p_{2}(2 R) / s}\left(\int_{B^{+}(2 R)}\left(1+|D h|^{2}\right)^{s / 2} d x\right)^{p_{2}(2 R) / s}\right] .
\end{aligned}
$$

Proof. In this proof, we abbreviate $p_{2}(2 R)$ to $p_{2}$. Let us define a frozen functional as

$$
\mathcal{D}_{0}(w):=\int_{B^{+}(R)}|D w|^{p_{2}} d x
$$

and let $w \in W^{1, p_{2}}\left(B^{+}(R)\right)$ be a minimizer of $\mathcal{D}_{0}$ with $w=v$ on $\partial B^{+}(R)$.

Since we are supposing (2.15), by virtue of Proposition 2.2, we see that $v \in W^{1,(1+\delta) p(x)}\left(B^{+}(R)\right) \subset$ $W^{1,(1+\delta / 2) p_{2}}\left(B^{+}(R)\right)$. So, using Corollary 2.3 with $D=B^{+}(R)$ and $S=R / k$ for a suitable $k>0$, we have

$$
\int_{B^{+}(R)}|D w|^{(1+\delta / 2) p_{2}} d x \leq c \int_{B^{+}(R)}\left(1+|D u|^{2}\right)^{(1+\delta / 2) p_{2} / 2} d x .
$$


On the other hand, by boundary regularity results for minimizers of functionals of standard growth (see for example $[6$, p. 446, 1.-5]), we have for any $k \in(0,1)$

$$
\int_{B_{\rho}^{+}}|D w|^{p_{2}} d x \leq c\left[\left(\left(\frac{\rho}{R}\right)^{m}+k\right) \int_{B^{+}(R)}|D w|^{p_{2}} d x+k^{1-p_{2}} R^{m\left(1-p_{2} / s\right)}\left(\int_{B^{+}(2 R)}|D h|^{s} d x\right)^{p_{2} / s}\right] .
$$

As in $[4,(9)]$, the minimality of $v$ implies that

$$
\mathcal{D}_{0}(v)-\mathcal{D}_{0}(w) \geq c \int_{B^{+}(R)}(|D v|+|D w|)^{p_{2}-2}|D v-D w|^{2} d x .
$$

(Although in [4] the integrand is $(|D u|-|D v|)^{p_{2}-2} \ldots$, the minus sign in the parentheses is clearly a typo.) So, we have

$$
\int_{B^{+}(R)}|D v-D w|^{p_{2}} d x \leq \mathcal{D}_{0}(v)-\mathcal{D}_{0}(w) .
$$

Since $v$ minimize $\mathcal{D}_{p(x)}$,

$$
\mathcal{D}_{0}(v)-\mathcal{D}_{0}(w) \leq \mathcal{D}_{0}(v)-\mathcal{D}_{p(x)}(v)+\mathcal{D}_{p(x)}(w)-\mathcal{D}_{0}(w) .
$$

In order to estimate the right-hand side of the above inequality, we mention that as (7) in [4] for every $\varepsilon>0$ there exists a constant $C(\varepsilon)>0$ such that

$$
\left|t^{r}-t^{s}\right| \leq C(\varepsilon)(s-r)\left(1+t^{(1+\varepsilon) s}\right), \quad \text { for all } t \geq 0 \text { and } s \geq r \geq 1 .
$$

By virtue of the above inequality, Hölder continuity of $p(x)$, and the assumption (2.15), using Proposition 2.2, we can estimate as follows:

$$
\begin{aligned}
& \left|\mathcal{D}_{0}(v)-\mathcal{D}_{p(x)}(v)\right| \\
& \leq c R^{\sigma} \int_{B^{+}(R)}\left(1+|D v|^{2}\right)^{(1+\delta / 2) p_{2} / 2} d x \\
& \leq c 7 R^{\sigma} \int_{B^{+}(R)}\left(1+|D v|^{2}\right)^{(1+\delta) p(x) / 2} d x \\
& \leq c R^{\sigma-m \delta}\left(\int_{B^{+}(2 R)}\left(1+|D v|^{2}\right)^{p(x) / 2} d x\right)^{1+\delta}+c R^{\sigma} \int_{B^{+}(2 R)}\left(1+|D h|^{2}\right)^{(1+\delta) p(x) / 2} d x \\
& \leq c R^{\sigma-m \delta} \int_{B^{+}(2 R)}\left(1+|D v|^{2}\right)^{p(x) / 2} d x+c R^{\sigma+m\left(1-(1+\delta) p_{2} / s\right)}\left(\int_{B^{+}(2 R)}\left(1+|D h|^{2}\right)^{s / 2} d x\right)^{(1+\delta) p_{2} / s} .
\end{aligned}
$$

Here, we used the assumption $\int|D v|^{p(x)} d x \leq K$ for the last inequality.

Using Corollary 2.3 with $p(x)=p_{2}, v=w$ and $h=v$ and the latter half of the above estimates, we can estimate $\left|\mathcal{D}_{p(x)}(w)-\mathcal{D}_{0}(w)\right|$ similarly.

$$
\begin{aligned}
& \left|\mathcal{D}_{p(x)}(w)-\mathcal{D}_{0}(w)\right| \\
& \leq c R^{\sigma} \int_{B^{+}(R)}\left(1+|D w|^{2}\right)^{(1+\delta / 2) p_{2} / 2} d x \\
& \leq c R^{\sigma} \int_{B^{+}(R)}\left(1+|D v|^{2}\right)^{(1+\delta / 2) p_{2} / 2} d x \\
& \leq c R^{\sigma-m \delta} \int_{B^{+}(2 R)}\left(1+|D v|^{2}\right)^{p(x) / 2} d x+c R^{\sigma+m\left(1-(1+\delta) p_{2} / s\right)}\left(\int_{B^{+}(2 R)}\left(1+|D h|^{2}\right)^{s / 2} d x\right)^{(1+\delta) p_{2} / s}
\end{aligned}
$$


Combining (2.20), (2.21), (2.23) and (2.24), we get

$$
\begin{aligned}
& \int_{B^{+}(R)}|D v-D w|^{p_{2}} d x \\
& \quad \leq c R^{\sigma-m \delta} \int_{B^{+}(2 R)}\left(1+|D v|^{2}\right)^{p(x) / 2} d x+c R^{\sigma+m\left(\sigma-(1+\delta) p_{2} / s\right)}\left(\int_{B^{+}(2 R)}\left(1+|D h|^{2}\right)^{s / 2} d x\right)^{(1+\delta) p_{2} / s} .
\end{aligned}
$$

Combining (2.19) with the above estimate, we obtain

$$
\begin{aligned}
& \int_{B^{+}(\rho)}|D v|^{p_{2}} d x \\
& \leq c\left[\left(\frac{\rho}{R}\right)^{m}+k\right] \int_{B^{+}(2 R)}|D w|^{p_{2}} d x+c k^{1-p_{2}} R^{m\left(1-p_{2} / s\right)}\left(\int_{B^{+}(R)}|D h|^{s} d x\right)^{p_{2} / s} \\
& \quad+c R^{\sigma-m \delta} \int_{B^{+}(2 R)}\left(1+|D v|^{2}\right)^{p(x) / 2} d x+c R^{\sigma+m\left(1-(1+\delta) p_{2} / s\right)}\left(\int_{B^{+}(2 R)}\left(1+|D h|^{2}\right)^{s / 2} d x\right)^{(1+\delta) p_{2} / s} .
\end{aligned}
$$

So, taking $R \leq 1$ sufficiently small so that $\int_{B^{+}(2 R)}\left(1+|D h|^{2}\right)^{s / 2} d x \leq 1$, and remarking that $\sigma-m(1+\delta) p_{2} / s \geq$ $-m p_{2} / s$, and using the minimality of $w$, we see that

$$
\begin{aligned}
& \int_{B^{+}(\rho)}|D v|^{p_{2}} d x \\
& \quad \leq c\left[\left(\frac{\rho}{R}\right)^{m}+k+R^{\sigma-m \delta}\right] \int_{B^{+}(2 R)}|D v|^{p_{2}} d x+c\left(k^{1-p_{2}}+1\right) R^{m-p_{2} m / s}\left(\int_{B^{+}(2 R)}\left(1+|D h|^{2}\right)^{s / 2} d x\right)^{p_{2} / s}
\end{aligned}
$$

holds. Now, by virtue of a well-known lemma (see, for example [10, Lemma 5.13] taking $k$ and $R$ sufficiently small, we get the decay estimate (2.16).

\section{Partial regularity up to the boundary}

In this section we consider the boundary analogue of the result of [15].

For a map $u: \Omega \rightarrow \mathbb{R}^{n}$ under consideration, we introduce the following quantities:

$$
\begin{aligned}
& \Phi(x, r, p):=r\left(r^{-m} \int_{\Omega(x, r)}\left(1+|D u(y)|^{2}\right)^{p / 2} d y\right)^{1 / p}, \\
& \Psi(x, r):=\Phi\left(x, r, p_{2}(x, r)\right) .
\end{aligned}
$$

For these quantities we prepare the following simple estimates.

Lemma 3.1. For $\gamma_{1} \leq p<q \leq \gamma_{2}$, we have

$$
\Phi(x, r, p) \leq \omega_{m}^{\left(1 / \gamma_{1}\right)-\left(1 / \gamma_{2}\right)} \Phi(x, r, q),
$$

where $\omega_{m}$ stands for the volume of the $m$-dimensional unit ball.

For some $x, x^{\prime} \in \Omega, r>0$ and $k>1$, suppose that $B(x, r) \subset B\left(x^{\prime}, k r\right)$, then we see that

$$
\Psi(x, r) \leq \omega_{m}^{\left(1 / \gamma_{1}\right)-\left(1 / \gamma_{2}\right)} k^{\left(m / \gamma_{1}\right)-1} \Psi\left(x^{\prime}, k r\right) .
$$


Proof. Using Hölder's inequality, we see that

$$
\begin{aligned}
\Psi(x, r, p) & =r\left(r^{-m} \int_{\Omega(x, r)}\left(1+|D u|^{2}\right)^{p / 2} d y\right)^{1 / p} \\
& \leq r\left[r^{-m}\left(\int_{B(x, r)} 1 d y\right)^{1-p / q}\left(\int_{\Omega(x, r)}\left(1+|D u|^{2}\right)^{q / 2} d x\right)^{p / q}\right]^{1 / p} \\
& \leq r\left[r^{-m}\left(\omega_{m} r^{m}\right)^{1-p / q}\left(\int_{\Omega(x, r)}\left(1+|D u|^{2}\right)^{q / 2} d y\right)^{p / q}\right]^{1 / p} \\
& =\omega_{m}^{(1 / p)-(1 / q)} r\left(r^{-m} \int_{\Omega(x, r)}\left(1+|D u|^{2}\right)^{q / 2} d y\right)^{1 / q} \\
& =\omega_{m}^{\left(1 / \gamma_{1}\right)-\left(1 / \gamma_{2}\right)} \Phi(x, r, q),
\end{aligned}
$$

where we also used the fact that $\omega_{m}>1$. Thus we get (3.3).

Since the inclusion $B(x, r) \subset B\left(x^{\prime}, k r\right)$ implies that $p_{2}(x, r) \leq p_{2}\left(x^{\prime}, k r\right)$, using (3.3), we see that

$$
\begin{aligned}
\Phi(x, r) & \leq\left(k^{m-p_{2}(x, r)}(k r)^{p_{2}(x, r)-m} \int_{\Omega\left(x^{\prime}, k r\right)}\left(1+|D u|^{2}\right)^{p_{2}(x, r)} d x\right)^{1 / p_{2}(x, r)} \\
& =k^{\left(m / p_{2}(x, r)\right)-1} \Phi\left(x^{\prime}, k r, p_{2}(x, r)\right) \\
& \leq k^{\left(m / p_{2}(x, r)\right)-1} \omega_{m}^{\left(1 / \gamma_{1}\right)-\left(1 / \gamma_{2}\right)} \Psi\left(x^{\prime}, k r\right) \\
& \leq k^{\left(m / \gamma_{1}\right)-1} \omega_{m}^{\left(1 / \gamma_{1}\right)-\left(1 / \gamma_{2}\right)} \Psi\left(x^{\prime}, k r\right) .
\end{aligned}
$$

Thus we get (3.4).

In the following we abbreviate

$$
C_{*}:=\omega_{m}^{\left(1 / \gamma_{1}\right)-\left(1 / \gamma_{2}\right)}, \quad \gamma_{3}:=\frac{m}{\gamma_{1}}-1
$$

Theorem 3.2. Let $R_{1}>R_{2}$ be positive constants. Assume that $g^{\alpha \beta}, G_{i j}(u)$ and $p(x)$ satisfy the conditions $(C 1)-(C 3)$ in $B^{+}\left(R_{1}\right)=B^{+}\left(0, R_{1}\right)$, and that

$$
\omega_{p}\left(R_{1}\right) \leq \delta, \quad\left(1+\frac{\delta}{2}\right) p_{2}\left(R_{1}\right) \leq(1+\delta) p_{1}\left(R_{1}\right)
$$

(For the constant $\delta$ see the comments before (2.13).) Let $u \in W^{1, p(x)}\left(B^{+}\left(R_{1}\right)\right)$ be a local minimizer of the functional $\mathcal{E}\left(v ; B^{+}\left(R_{1}\right)\right)$ in the class

$$
\left\{v \in W^{1, p(x)} ; v=h \text { on } \Gamma\left(R_{1}\right)\right\},
$$

for a given boundary data $h \in W^{1, s}\left(B^{+}\left(R_{1}\right)\right)$ with $s>(1+\delta) \max \left\{m, \gamma_{2}\right\}$.

Then, there exist positive constants $r_{1}$ and $\varepsilon_{0}$ with the following property: if for some $x \in B^{+}\left(R_{2}\right)$ and $r_{0} \in\left(0, r_{1}\right)$ we have $\Psi\left(x, r_{0}\right) \leq \varepsilon_{0}$, then $u$ satisfies for some $\alpha \in(0,1)$

$$
\Psi(x, \rho) \leq c \rho^{\alpha}, \quad \text { for any } \rho \in\left(0, r_{0}\right) .
$$

Proof. Take a point $x_{1} \in \Gamma\left(R_{2}\right)$ arbitrarily. For $r<R_{1}-R_{2}$ let us put

$$
\begin{aligned}
& B_{r}^{+}:=B^{+}\left(x_{1}, r\right), \\
& p_{1}(r):=p_{1}\left(x_{1}, r\right)=\inf _{B_{r}^{+}} p(x), \quad p_{2}(r):=p_{2}\left(x_{1}, r\right)=\sup _{B_{r}^{+}} p(x) .
\end{aligned}
$$


Let $R$ be a positive constant with $R \leq\left(R_{1}-R_{2}\right) / 2$. As in [15], we define two types of frozen functionals.

$$
\begin{aligned}
& \mathcal{F}_{1}(v):=\int_{B_{R}^{+}}\left(g_{R}^{\alpha \beta} G_{i j}\left(u_{R}\right) D_{\alpha} v^{i} D_{\beta} v^{j}\right)^{p(x) / 2} d x, \\
& \mathcal{F}_{2}(v):=\int_{B_{R}^{+}}\left(g_{R}^{\alpha \beta} G_{i j}\left(u_{R}\right) D_{\alpha} v^{i} D_{\beta} v^{j}\right)^{p_{2}(2 R) / 2} d x,
\end{aligned}
$$

where we are writing

$$
g_{R}^{\alpha \beta}=g_{x_{1}, R}^{\alpha \beta}:=\int_{B_{R}^{+}} g^{\alpha \beta}(x) d x \quad \text { and } \quad u_{R}=u_{x_{1}, R}:=\int_{B_{R}^{+}} u(x) d x .
$$

Let $v$ be a minimizer of $\mathcal{F}_{1}$ in the class

$$
u+W_{0}^{1, p(x)}\left(B_{R}^{+}\right):=\left\{w \in W^{1, p(x)}\left(B_{R}^{+}\right) ; w-u \in W_{0}^{1, p(x)}\left(B_{R}^{+}\right)\right\} .
$$

Then, using Corollary 2.3 with $D=B_{R}^{+}$and $h=u$, we have for any $\varepsilon \in(0, \delta]$

$$
\int_{B_{R}^{+}}\left(1+|D v|^{2}\right)^{(1+\varepsilon) p(x) / 2} d x \leq c \int_{B_{R}^{+}}\left(1+|D u|^{2}\right)^{(1+\varepsilon) p(x) / 2} d x .
$$

On the other hand, for any $\beta \in\left(0, m p_{2}(2 R) / s\right)$, from Theorem 2.6, we can see that the following estimate holds for any $0<\rho<R / 2$.

$$
\int_{B_{\rho}^{+}}|D v|^{p_{2}(2 R)} d x \leq c\left[\left(\frac{\rho}{R}\right)^{m-\beta} \int_{B_{R}^{+}}|D v|^{p_{2}(2 R)} d x+\rho^{m-m p_{2}(2 R) / s}\left(\int_{B_{2 R}^{+}}\left(1+|D h|^{2}\right)^{s / 2} d x\right)^{p_{2}(2 R) / s}\right] .
$$

Using (3.12) with $\varepsilon=\omega_{p}(2 R)\left(<\omega_{p}\left(2 R_{1}\right) \leq \delta\right)$ and Proposition 2.2, we can estimate the integral of the first term of the right hand side of (3.13) as

$$
\begin{aligned}
& \int_{B_{R}^{+}}|D v|^{p_{2}(2 R)} d x \\
& \leq c \int_{B_{R}^{+}}\left(1+|D v|^{2}\right)^{\left(1+\omega_{1}(2 R)\right) p(x) / 2} d x \\
& \leq c \int_{B_{R}^{+}}\left(1+|D u|^{2}\right)^{\left(1+\omega_{1}(2 R)\right) p(x) / 2} d x \\
& \leq c R^{-m \omega_{1}(2 R)}\left(\int_{B_{2 R}^{+}}\left(1+|D u|^{2}\right)^{p(x) / 2} d x\right)^{1+\omega_{1}(2 R)}+c \int_{B_{2 R}^{+}}\left(1+|D h|^{2}\right)^{\left(1+\omega_{1}(2 R)\right) p(x) / 2} d x \\
& \leq c R^{-m \omega_{1}(2 R)} \int_{B_{2 R}^{+}}\left(1+|D u|^{2}\right)^{p(x) / 2} d x+c \int_{B_{2 R}^{+}}\left(1+|D h|^{2}\right)^{(1+\delta) p_{2}(2 R) / 2} d x \\
& \leq c R^{-m \omega_{1}(2 R)} \int_{B_{2 R}^{+}}\left(1+|D u|^{2}\right)^{p_{2}(2 R) / 2} d x+c R^{m\left(1-(1+\delta) p_{2}(2 R) / s\right)}\left(\int_{B_{2 R}^{+}}\left(1+|D h|^{2}\right)^{s / 2} d x\right)^{(1+\delta) p_{2}(2 R) / s} .
\end{aligned}
$$

Here, we used (3.12) for the second inequality, Proposition 2.2 for the third and boundedness of $\int_{\Omega}\left(1+|D u|^{2}\right)^{p(x) / 2} d x$ for the fourth. In what follows, we abbreviate as $p_{2}=p_{2}(2 R)$ and $\bar{p}_{2}=(1+\delta) p_{2}(2 R)$. Since we see that $R^{-m \omega_{1}(2 R)}$ is bounded by virtue of (2.4), from (3.13) and the above estimate, we obtain for $\beta \in\left(0, m p_{2} / s\right)$ 


$$
\begin{aligned}
\int_{B_{\rho}^{+}}|D v|^{p_{2}} d x \leq & c\left(\frac{\rho}{R}\right)^{m-\beta} \int_{B_{2 R}^{+}}\left(1+|D u|^{2}\right)^{p_{2} / 2} d x \\
& +c \rho^{m-m p_{2} / s}\left(\int_{B_{2 R}^{+}}\left(1+|D h|^{2}\right)^{s / 2} d x\right)^{\bar{p}_{2} / s}+c \rho^{m-m p_{2} / s}\left(\int_{B_{2 R}^{+}}\left(1+|D h|^{2}\right)^{s / 2}\right)^{p_{2} / s} .
\end{aligned}
$$

Let us write

$$
K(h):=\left(\int_{B^{+}\left(R_{1}\right)}\left(1+|D h|^{2}\right)^{s / 2} d x\right)^{1 / s}, \quad \hat{K}(h):=\max \left\{K(h)^{p_{2}}, K(h)^{\bar{p}_{2}}\right\} .
$$

Then, from (3.14), we have for some positive constants $K_{1}$ and $K_{2}$ that

$$
\int_{B_{\rho}^{+}}|D v|^{p_{2}} d x \leq K_{1}\left(\frac{\rho}{R}\right)^{m-\beta} \int_{B_{2 R}^{+}}\left(1+|D u|^{2}\right)^{p_{2} / 2} d x+K_{2} \rho^{m-m \bar{p}_{2} / s} \hat{K}(h) .
$$

On the other hand, proceeding as in [15, pp. 3343-3344], we can estimate $\int_{B_{R}^{+}}|D u-D v|^{p_{2}(2 R)} d x$ as follows:

$$
\begin{aligned}
& \int_{B_{R}^{+}}|D u-D v|^{p_{2}(2 R)} d x \\
& \leq c_{6}\left(\mathcal{F}_{2}(u)-\mathcal{F}_{2}(v)\right)+c R^{\sigma} \int_{B_{R}^{+}}\left(1+|D v|^{2}\right)^{(1+\varepsilon) p_{2} / 2} d x \\
& \leq c_{5}\left(\mathcal{F}_{2}(u)-\mathcal{F}_{1}(u)+\mathcal{F}_{1}(u)-\mathcal{F}(u)+\mathcal{F}(u)-\mathcal{F}(v)\right. \\
&\left.+\mathcal{F}(v)-\mathcal{F}_{1}(v)+\mathcal{F}_{1}(v)-\mathcal{F}_{2}(v)\right)+c R^{\sigma} \int_{B_{R}^{+}}\left(1+|D v|^{2}\right)^{(1+\varepsilon) p_{2} / 2} d x \\
& c_{4}\left\{\left(\mathcal{F}_{2}(u)-\mathcal{F}_{1}(u)\right)+\left(\mathcal{F}_{1}(u)-\mathcal{F}(u)\right)+\left(\mathcal{F}(v)-\mathcal{F}_{1}(v)\right)\right. \\
&\left.+\left(\mathcal{F}_{1}(v)-\mathcal{F}_{2}(v)\right)\right\}+c R^{\sigma} \int_{B_{R}^{+}}\left(1+|D v|^{2}\right)^{(1+\varepsilon) p_{2} / 2} d x \\
&=: I+I I+I I I+I V+V .
\end{aligned}
$$

In order to estimate $|I|$ and $|I V|$, we use (2.22) with $s=p_{2}(2 R) / 2$ and $r=p(x) / 2$ and get

$$
\begin{aligned}
& |I| \leq c R^{\sigma} \int_{B_{R}^{+}}\left(1+|D u|^{2}\right)^{(1+\varepsilon) p_{2}(2 R) / 2} d x, \\
& |I V| \leq c R^{\sigma} \int_{B_{R}^{+}}\left(1+|D v|^{2}\right)^{(1+\varepsilon) p_{2}(2 R) / 2} d x .
\end{aligned}
$$

Let us take $\varepsilon<\delta / 2$, then by the assumption (3.6), we have

$$
(1+\varepsilon) p_{2}(2 R) \leq\left(1+\frac{\delta}{2}\right) p_{2}(2 R)<(1+\delta) p_{1}(2 R) \leq(1+\delta) p(x) .
$$

So, we can estimate $I$ as

$$
|I| \leq c R^{\sigma} \int_{B_{R}^{+}}\left(1+|D u|^{2}\right)^{(1+\delta) p(x) / 2} d x .
$$


Using Corollary 2.3, we have

$$
\begin{aligned}
|I V|,|V| & \leq c R^{\sigma} \int_{B_{R}^{+}}\left(1+|D v|^{2}\right)^{(1+\delta) p(x) / 2} d x \\
& \leq c R^{\sigma} \int_{B_{R}^{+}}\left(1+|D u|^{2}\right)^{(1+\delta) p(x) / 2} d x .
\end{aligned}
$$

Using Proposition 2.2 we can see that

$$
\begin{aligned}
& R^{\sigma} \int_{B_{R}^{+}}\left(1+|D u|^{2}\right)^{(1+\delta) p(x) / 2} d x \\
& \leq c R^{\sigma-m \delta}\left(\int_{B_{2 R}^{+}}\left(1+|D u|^{2}\right)^{p(x) / 2} d x\right)^{1+\delta}+c R^{\sigma} \int_{B_{2 R}^{+}}\left(1+|D h|^{2}\right)^{p(x) / 2} d x \\
& \quad \leq c R^{\sigma-m \delta} \int_{B_{2 R}^{+}}\left(1+|D u|^{2}\right)^{p(x) / 2} d x+c R^{\sigma+m-m p_{2} / s} K(h)^{p_{2}},
\end{aligned}
$$

where we used the boundedness of $\int|D u|^{p(x)} d x$. Combining (3.19) and (3.20) with (3.21), we obtain

$$
|I|,|I V|,|V| \leq c R^{\sigma-m \delta}\left[\int_{B_{2 R}^{+}}\left(1+|D u|^{2}\right)^{p(x) / 2} d x+c R^{m-m p_{2} / s} K(h)^{p_{2}}\right],
$$

where we used the fact that $R^{\sigma}<R^{\sigma-m \delta}$.

Let us estimate $|I I+I I I|$. Writing $q=1+\delta$ and $q^{\prime}=q /(q-1)=(1+\delta) / \delta$, using Hölder's inequality, and remembering the condition $(\mathrm{C} 2)$, we have

$$
\begin{aligned}
|I I+I I I| \leq & c\left[\left(\int_{B_{R}^{+}} \omega_{G}^{q^{\prime}}\left(\left|u-u_{R}\right|^{2}\right) d x\right)^{1 / q^{\prime}}+\left(\int_{B_{R}^{+}}\left|g(x)-g_{R}\right|^{q^{\prime}} d x\right)^{1 / q^{\prime}}\right]\left(\int_{B_{R}^{+}}|D u|^{q p(x)} d x\right)^{1 / q} \\
& +c\left[\left(\int_{B_{R}^{+}} \omega_{G}^{q^{\prime}}\left(\left|v-u_{R}\right|^{2}\right) d x\right)^{1 / q^{\prime}}+\left(\int\left|g(x)-g_{R}\right|^{q^{\prime}} d x\right)^{1 / q^{\prime}}\right]\left(\int_{B_{R}^{+}}|D v|^{q p(x)} d x\right)^{1 / q} \\
= & : c\left(I I^{\prime}+I I I^{\prime}\right) .
\end{aligned}
$$

Here and in the sequel, we write

$$
g(x)=\left(g^{\alpha \beta}(x)\right), \quad \text { and } \quad\left|g(x)-g_{R}\right|=\left\{\sum_{\alpha \beta}\left(g^{\alpha \beta}(x)-g_{R}^{\alpha \beta}\right)^{2}\right\}^{1 / 2} .
$$

Since $\omega_{G}$ and $g$ are bounded, using Proposition 2.2, Jensen's inequality, Hölder's inequality and the SobolevPoincaré inequality, we can estimate $I I^{\prime}$ as follows:

$$
\begin{aligned}
I I^{\prime} \leq & c\left[\omega_{G}^{1 / q^{\prime}}\left(c R^{2-m} \int_{B_{R}^{+}}|D u|^{2} d x\right)+\left(f_{B_{R}^{+}}\left|g(x)-g_{R}\right| d x\right)^{1 / q^{\prime}}\right] \\
& \times\left[\int_{B_{2 R}^{+}}\left(1+|D u|^{2}\right)^{p(x) / 2} d x+\left(R^{\delta m} \int_{B_{2 R}^{+}}\left(1+|D h|^{2}\right)^{q p(x) / 2} d x\right)^{1 / q}\right]
\end{aligned}
$$




$$
\begin{aligned}
\leq & c\left[\omega_{G}^{1 / q^{\prime}}\left(c\left\{R^{p_{2}(2 R)-m} \int_{B_{R}^{+}}|D u|^{p_{2}} d x\right\}^{2 / p_{2}}\right)+c \omega_{g}(2 R)^{1 / q^{\prime}}\right] \\
& \times\left[\int_{B_{2 R}^{+}}\left(1+|D u|^{2}\right)^{p(x) / 2} d x+\left(R^{\delta m} \int_{B_{2 R}^{+}}\left(1+|D h|^{2}\right)^{\bar{p}_{2} / 2} d x\right)^{1 / q}\right] \\
\leq & c\left[\omega_{G}^{1 / q^{\prime}}\left(c\left\{R^{p_{2}(2 R)-m} \int_{B_{R}^{+}}|D u|^{p_{2}} d x\right\}^{2 / p_{2}}\right)+c \omega_{g}^{1 / q^{\prime}}\right] \\
& \times\left[\int_{B_{2 R}^{+}}\left(1+|D u|^{2}\right)^{p(x) / 2} d x+R^{m\left(1-p_{2} / s\right)} K(h)^{p_{2}}\right] .
\end{aligned}
$$

Using (3.12) and proceeding as above, we estimate $I I^{\prime}$ as

$$
\begin{aligned}
I I I^{\prime} \leq & c\left[\left(\int_{B_{R}^{+}} \omega_{G}\left(\left|u-u_{R}\right|^{2}+|u-v|^{2}\right) d x\right)^{1 / q^{\prime}}+\left(\int_{B_{R}^{+}}\left|g(x)-g_{R}\right| d x\right)^{1 / q^{\prime}}\right]\left(\int_{B_{R}^{+}}\left(1+|D u|^{2}\right)^{q p(x) / 2} d x\right)^{1 / q} \\
\leq & c\left[\omega_{G}^{1 / q^{\prime}}\left(f_{B_{R}^{+}}\left|u-u_{R}\right|^{2} d x+f_{B_{R}^{+}}|u-v|^{2} d x\right)\right. \\
& \left.+\left(f\left|g(x)-g_{R}\right| d x\right)^{1 / q^{\prime}}\right]\left[\int_{B_{2 R}^{+}}\left(1+|D u|^{2}\right)^{p(x) / 2} d x+\left(R^{\delta m} \int_{B_{2 R}^{+}}\left(1+|D h|^{2}\right)^{q p(x) / 2} d x\right)^{1 / q}\right] \\
\leq & c\left[\omega_{G}^{1 / q^{\prime}}\left(c R^{2-m} \int_{B_{R}^{+}}|D u|^{2} d x+c R^{2-m} \int_{B_{R}^{+}}|D v|^{2} d x\right)\right. \\
& \left.+\left(f\left|g(x)-g_{R}\right| d x\right)^{1 / q^{\prime}}\right]\left[\int_{B_{2 R}^{+}}\left(1+|D u|^{2}\right)^{p(x) / 2} d x+R^{m\left(1-p_{2} / s\right)} K(h)^{p_{2}}\right] .
\end{aligned}
$$

Again with (3.12) and Proposition 2.2, we can estimate the second term in $\omega_{G}$ as follows:

$$
\begin{aligned}
R^{2-m} \int_{B_{R}^{+}}|D v|^{2} d x \leq & c\left(R^{p_{2}(2 R)-m} \int_{B_{R}^{+}}|D v|^{p_{2}(2 R)} d x\right)^{2 / p_{2}} \\
\leq & c\left(R^{p_{2}-m} \int_{B_{R}^{+}}\left(1+|D v|^{2}\right)^{\left(1+\omega_{p}(2 R)\right) p(x) / 2} d x\right)^{2 / p_{2}} \\
\leq & c\left(R^{p_{2}-m} \int_{B_{R}^{+}}\left(1+|D u|^{2}\right)^{\left(1+\omega_{p}(2 R)\right) p(x) / 2} d x\right)^{2 / p_{2}} \\
\leq & c\left[R ^ { p _ { 2 } - m } \left\{R^{-\omega_{p}(2 R) m}\left(\int_{B_{2 R}^{+}}\left(1+|D u|^{2}\right)^{p(x) / 2} d x\right)^{1+\omega_{p}(2 R)}\right.\right. \\
& \left.\left.+\int_{B_{2 R}^{+}}\left(1+|D h|^{2}\right)^{\left(1+\omega_{p}(2 R)\right) p(x) d x}\right\}\right]^{2 / p_{2}},
\end{aligned}
$$

where we used Proposition 2.2 with $\delta=\omega_{p}(2 R)$ for the fourth inequality. Since $R^{-\omega_{1}(2 R)}$ and $\int|D u|^{p(x)} d x$ are bounded, from the above estimate, we obtain 


$$
\begin{aligned}
& R^{2-m} \int_{B_{R}^{+}}|D v|^{2} d x \\
& \leq c\left(R^{p_{2}-m} \int_{B_{2 R}^{+}}\left(1+|D u|^{2}\right)^{p(x) / 2} d x\right)^{2 / p_{2}}+c\left(R^{p_{2}-m} \int_{B_{2 R}^{+}}\left(1+|D h|^{2}\right)^{\bar{\rho}_{2}} d x\right)^{2 / p_{2}} \\
& \quad \leq c\left(R^{p_{2}-m} \int_{B_{2 R}^{+}}\left(1+|D u|^{2}\right)^{p_{2} / 2} d x\right)^{2 / p_{2}}+c\left[R^{p_{2}-m} R^{m-m p_{2} / s}\left(\int_{B_{2 R}^{+}}\left(1+|D h|^{2}\right)^{s} d x\right)^{\bar{p}_{2} / s}\right]^{2 / p_{2}} \\
& \quad \leq c\left(R^{p_{2}-m} \int_{B_{2 R}^{+}}\left(1+|D u|^{2}\right)^{p_{2} / 2} d x\right)^{2 / p_{2}}+c R^{2(1-q m / s)} K(h)^{2 q} .
\end{aligned}
$$

Here, mention that by the assumption that $s>(1+\delta) m$ we have $1-m q / s=1-m(1+\delta) / s>0$.

From (3.25) and (3.26) we obtain

$$
\begin{aligned}
I I I^{\prime} \leq & c\left[\omega_{G}^{1 / q^{\prime}}\left(c_{8} \Psi(2 R)^{2}+c_{9} R^{2(1-q m / s)} K(h)^{2 q}\right)+\omega_{g}^{1 / q^{\prime}}(2 R)\right] \\
\times & {\left[\int_{B_{2 R}^{+}}\left(1+|D u|^{2}\right)^{p(x) / 2} d x+R^{m\left(1-p_{2} / s\right)} K(h)^{p_{2}}\right] . }
\end{aligned}
$$

Now, combining (3.17), (3.22), (3.23), (3.24) and (3.27), we obtain

$$
\begin{array}{rl}
\int_{B_{R}^{+}} \mid D & u-\left.D v\right|^{p_{2}} d x \\
\leq & c R^{\sigma-m \delta}\left[\int_{B_{2 R}^{+}}\left(1+|D u|^{2}\right)^{p_{2} / 2}+R^{m\left(1+\delta-p_{2} / s\right)} K(h)^{p_{2}}\right] \\
+ & c\left[\omega_{G}^{1 / q^{\prime}}\left(c_{8} \Psi(2 R)^{2}+c_{9} R^{2(1-q m / s)} K(h)^{2 q}\right)+\omega_{g}^{1 / q^{\prime}}(2 R)\right] \\
\times & {\left[\int_{B_{2 R}^{+}}\left(1+|D u|^{2}\right)^{p(x) / 2} d x+R^{m\left(1-p_{2} / s\right)} K(h)^{p_{2}}\right]} \\
\leq & c\left[R^{\sigma-m \delta}+\omega_{G}^{1 / q^{\prime}}\left(c_{8} \Psi(2 R)^{2}+c_{9} R^{2(1-q m / s)} K(h)^{2 q}\right)+\omega_{g}^{1 / q^{\prime}}(2 R)\right] \\
\times & {\left[\int_{B_{2 R}^{+}}\left(1+|D u|^{2}\right)^{p(x) / 2} d x+R^{m\left(1-p_{2} / s\right)} K(h)^{p_{2}}\right] .}
\end{array}
$$

Here, for the last inequality, we used the fact that $R^{\delta}<1$.

Now, putting $r=2 R, \hat{\omega}_{G}(t)=\omega_{G}^{1 / q^{\prime}}\left(\max \left\{c_{8}, c_{9}\right\} \cdot t\right)$ and $\hat{\omega}_{g}=\omega_{g}^{1 / q^{\prime}}$, from (3.16) and (3.28) we obtain

$$
\begin{aligned}
\int_{B_{\rho}^{+}} & \left(1+|D u|^{2}\right)^{p_{2} / 2} d x \\
\leq & K_{1}\left(\frac{\rho}{r}\right)^{m-\beta} \int_{B_{r}^{+}}\left(1+|D u|^{2}\right)^{p_{2} / 2} d x+K_{2} \rho^{m\left(1-\bar{p}_{2} / s\right)} \hat{K}(h) \\
& +c\left[r^{\sigma-m \delta}+\hat{\omega}_{G}+\hat{\omega}_{g}\right] \times\left[\int_{B_{r}^{+}}\left(1+|D u|^{2}\right)^{p_{2} / 2} d x+r^{m\left(1-p_{2} / s\right)} K(h)^{p_{2}}\right]
\end{aligned}
$$




$$
\begin{aligned}
\leq & K_{3}\left[\left(\frac{\rho}{r}\right)^{m-\beta}+r^{\sigma-m \delta}+\hat{\omega}_{G}+\hat{\omega}_{g}\right] \int_{B_{r}^{+}}\left(1+|D u|^{2}\right)^{p_{2} / 2} d x \\
& +K_{4}\left[1+r^{\sigma-m \delta}+\hat{\omega}_{G}+\hat{\omega}_{g}\right] r^{m\left(1-\bar{p}_{2} / s\right)} \hat{K}(h),
\end{aligned}
$$

for some constants $K_{3}$ and $K_{4}$. Here, for the second inequality we used the fact that $r<1$ and $\bar{p}_{2}>p_{2}$.

For $\tau \in(0,1)$ which will be specified later, put $\rho=\tau r$ in the above estimate and multiply both sides by $(\tau r)^{p_{2}-m}$, then we have

$$
\begin{aligned}
& (\tau r)^{p_{2}-m} \int_{B_{\tau r}^{+}}\left(1+|D u|^{2}\right)^{p_{2} / 2} d x \\
& \leq K_{3}\left[\tau^{p_{2}-\beta}+\tau^{p_{2}-m} r^{\sigma-m \delta}+\tau^{p_{2}-m} \hat{\omega}_{G}+\tau^{p_{2}-m} \hat{\omega}_{g}\right] r^{p_{2}-m} \int_{B_{r}^{+}}\left(1+|D u|^{2}\right)^{p_{2} / 2} d x \\
& \quad+K_{4}\left[\tau^{p_{2}-m}+\tau^{p_{2}-m} r^{\sigma-m \delta}+\tau^{p_{2}-m} \hat{\omega}_{G}+\tau^{p_{2}-m} \hat{\omega}_{g}\right] r^{p_{2}-m \bar{p}_{2} / s} \hat{K}(h) .
\end{aligned}
$$

Remembering the definitions of $\Phi$ and $\Psi$, and mentioning (3.3) and (3.5), from the above estimate we get

$$
\begin{aligned}
\left(\Psi\left(x_{1}, \tau r\right)\right)^{p_{2}(r)}= & \Phi\left(x_{1}, \tau r, p_{2}(\tau r)\right)^{p_{2}(r)} \\
\leq & C_{*}^{p_{2}(r)} \Phi\left(x_{1}, \tau r, p_{2}(r)\right)^{p_{2}(r)} \\
\leq & K_{3}^{p_{2}(r)} C_{*}^{p_{2}(r)}\left[\tau^{p_{2}-\beta}+\tau^{p_{2}-m}\left\{r^{\sigma-m \delta}\right.\right. \\
& \left.\left.+\hat{\omega}_{G}\left(\Psi\left(x_{1}, r\right)^{2}+r^{2(1-q / s)} K(h)^{2 q}\right)+\hat{\omega}_{g}(r)\right\}\right] \times \Phi\left(x_{1}, r\right)^{p_{2}} \\
& +\tau^{p_{2}-m} r^{p_{2}-m \bar{p}_{2} / s} C(g, G, p, h) \\
= & K_{3}^{p_{2}(r)} C_{*}^{p_{2}(r)} \tau^{p_{2}-\beta}\left[1+\tau^{\beta-m}\left\{r^{\sigma-m \delta}\right.\right. \\
& \left.\left.+\hat{\omega}_{G}\left(\Psi\left(x_{1}, r\right)^{2}+r^{2(1-q / s)} K(h)^{2 q}\right)+\hat{\omega}_{g}(r)\right\}\right] \times \Phi\left(x_{1}, r\right)^{p_{2}} \\
& +\tau^{p_{2}-m} r^{p_{2}-m \bar{p}_{2} / s} C(g, G, p, h),
\end{aligned}
$$

where $C(g, G, p, h)$ is a positive constant depending only on $g^{\alpha \beta}(x), G_{i j}(u), p(x)$ and $h(x)$. So, we obtain

$$
\begin{aligned}
\Psi\left(x_{1}, \tau r\right)= & K_{5} \tau^{1-\beta / p_{2}}\left[1+\tau^{(\beta-m) / p_{2}}\left\{r^{(\sigma-m \delta) / p_{2}}\right.\right. \\
& \left.\left.+\hat{\omega}_{G}^{1 / p_{2}}\left(\Psi\left(x_{1}, r\right)^{2}+r^{2(1-q / s)} K(h)^{2 q}\right)+\hat{\omega}_{g}^{1 / p_{2}}(r)\right\}\right] \times \Phi\left(x_{1}, r\right) \\
& +\tau^{1-m / p_{2}} r^{1-m q / s} C_{0}(g, G, p, h),
\end{aligned}
$$

where $K_{5}=K_{3} C_{*}$ and $C_{0}(g, G, p, h)=C(g, G, p, h)^{1 / p_{2}}$.

Since $0<\beta<1, m>2, \gamma_{1} \leq p_{2}=p_{2}(r) \leq \gamma_{2}$, and $\tau<1$, we have

$$
\tau^{(\beta-m) / p_{2}(r)} \leq \tau^{(\beta-m) / \gamma_{1}} .
$$

Without loss of generality we can assume that $0<r<1$, so we see that

$$
r^{(\sigma-m \delta) / p_{2}(r)} \leq r^{(\sigma-m \delta) / \gamma_{2}}, \quad(\tau r)^{1-\left(\beta / p_{2}(r)\right)} \leq(\tau r)^{1-\left(\beta / \gamma_{1}\right)} .
$$

In the following, since we consider the case that $\omega_{G}$ and $\omega_{g}$ are sufficiently small, we can assume that $\omega_{G}, \omega_{g}<1$. So, we have

$$
\hat{\omega}_{G}^{1 / p_{2}(r)} \leq \hat{\omega}_{G}^{1 / \gamma_{2}}, \quad \hat{\omega}_{g}^{1 / p_{2}(r)} \leq \hat{\omega}_{g}^{1 / \gamma_{2}} .
$$

For the sake of simplicity, let us put

$$
\mu_{1}:=1-\frac{\beta}{\gamma_{1}}, \quad \mu_{2}:=1-\frac{m q}{s}, \quad \tilde{\omega}_{G}:=\hat{\omega}_{G}^{1 / \gamma_{2}}, \quad \tilde{\omega}_{g}:=\hat{\omega}_{g}^{1 / \gamma_{2}} .
$$

Then, from (3.32), assuming $\Psi(r)<1$, we get 


$$
\begin{aligned}
\Psi(\tau r) \leq & K_{5} \tau^{\mu_{1}}\left[1+\tau^{(\beta-m) / \gamma_{1}}\left\{r^{(\sigma-m \delta) / \gamma_{2}}+\tilde{\omega}_{G}\left(\Psi(r)+r^{2 \mu_{2}} K(h)^{2 q}\right)+\tilde{\omega}_{g}\right\}\right] \Psi(r) \\
& +\tau^{1-m / p_{2}} r^{\mu_{2}} C_{0}(g, G, p, h)
\end{aligned}
$$

Now, let us take $\beta<m \gamma_{1} / s$, then we have $\mu_{1}=1-\beta / \gamma_{1}>1-q m / s=\mu_{2}$. Fix $v \in\left(\mu_{2}, \mu_{1}\right)$ and choose $\tau \in(0,1)$ so that $K_{5} \tau^{\mu_{1}} \leq \tau^{\nu} / 5$. Take $\varepsilon_{1}>0$ such that

$$
\tilde{\omega}_{G}\left(2 \varepsilon_{1}\right)<\tau^{(m-\beta) / \gamma_{1}} .
$$

Finally, let $r_{0}>0$ be a sufficiently small constant for which the following inequalities hold:

$$
\begin{aligned}
& \tau^{(\beta-m) \gamma_{1}} r_{0}^{(\sigma-m \delta) / \gamma_{2}}, \quad \tau^{(\beta-m) / \gamma_{1}} \tilde{\omega}_{g}\left(r_{0}\right) \leq 1 \\
& \tau^{1-m / \gamma_{1}} r_{0}^{\mu_{2}} C_{0}(g, G, p, h) \leq \varepsilon_{1} / 5, \quad r_{0}^{2 \mu_{2}} K(h)^{2 q} \leq \varepsilon_{1} .
\end{aligned}
$$

Now, assume that $\Psi\left(x_{1}, r\right) \leq \varepsilon_{1}$ for some $r \in\left(0, r_{0}\right)$, we obtain from (3.36)

$$
\begin{aligned}
\Psi\left(x_{1}, \tau r\right) & \leq \frac{\tau^{\nu}}{5}[1+1+1+1] \Psi\left(x_{1}, r\right)+\frac{\varepsilon_{1}}{5} \\
& =\frac{4}{5} \tau^{\nu} \Psi\left(x_{1}, r\right)+\frac{\varepsilon_{1}}{5} \\
& \leq \varepsilon_{0} .
\end{aligned}
$$

The above estimate enables us to use an iteration argument to get

$$
\begin{aligned}
\Psi\left(x_{1}, \tau^{k+1} r\right) & =\tau^{(k+1) \nu} \Psi\left(x_{1}, r\right)+C_{1} r^{\mu_{2}} \tau^{k \mu_{2}} \sum_{j=0}^{k} \tau^{\left(\nu-\mu_{2}\right) j} \\
& \leq \tau^{(k+1) \nu} \Psi\left(x_{1}, r\right)+C_{2}\left(\tau^{k} r\right)^{\mu_{2}},
\end{aligned}
$$

where $C_{1}=\tau^{1-m / \gamma_{1}} C_{0}$ and $C_{2}=C_{1} /\left(1-\tau^{\nu-\mu_{2}}\right)$.

For any $t \in(0, r)$, there exists a nonnegative integer $k$ such that $\tau^{k+1} r<t \leq \tau^{k} r$, and we have

$$
\begin{aligned}
\Psi\left(x_{1}, t\right) & \leq t\left(t^{-m}\left(\tau^{k} r\right)^{m}\left(\tau^{k} r\right)^{-m} \int_{B_{\tau^{k}}^{+}}\left(1+|D u|^{2}\right)^{p_{2}(t)} d x\right)^{1 / p_{2}(t)} \\
& \leq\left(\frac{\tau^{k} r}{t}\right)^{\left(m / p_{2}(t)\right)-1} \Phi\left(x_{1}, \tau^{k} r, p_{2}(t)\right) \\
& \leq C_{*}\left(\frac{\tau^{k} r}{t}\right)^{\left(m / p_{2}(t)\right)-1} \Psi\left(x, \tau^{k} r\right) \\
& \leq C_{*} \tau^{1-m / \gamma_{1}}\left(\tau^{-v} \tau^{(k+1) v} \Psi\left(x_{1}, r\right)+\tau^{-\mu_{2}} C_{2}\left(\tau^{k+1} r\right)^{\mu_{2}}\right) \\
& \leq C_{*} \tau^{1-\left(m / \gamma_{1}\right)-v}\left(\left(\frac{t}{r}\right)^{v} \Psi\left(x_{1}, r\right)+C_{2} \tau^{\mu_{2}}\right)
\end{aligned}
$$

For an interior point $x_{1} \in B^{+}\left(R_{2}\right)$ and for $0<t<r \leq \min \left\{R_{1}-R_{2}, x_{1}^{m}\right\}$, proceeding as above without the boundary condition $h$ or as in [15], we can get an estimate similar to (3.41). Consequently, we see that there are positive constants $r_{0} \in\left(0,\left(R_{1}-R_{2}\right) / 2\right), \varepsilon_{1}>0, \alpha \in(0,1), C_{A}$ and $C_{B}$ such that if

(a) $x_{1} \in \Gamma\left(R_{2}\right)$ and $\Psi\left(x_{1}, r\right) \leq \varepsilon_{1}$ for some $r \in\left(0, r_{0}\right)$,

or

(b) $x_{1} \in B^{+}\left(0, R_{2}\right), B\left(x_{1}, r\right) \in B^{+}\left(0, R_{1}\right)$ and $\Psi\left(x_{1}, r\right) \leq \varepsilon_{1}$ for some $r \in\left(0, r_{0}\right)$, 
then $\Psi\left(x_{1}, \rho\right)$ satisfies the following decay estimate:

$$
\Psi\left(x_{1}, t\right) \leq C_{A}\left(\frac{t}{r}\right)^{\alpha} \Psi(x, r)+C_{B} t^{\alpha} .
$$

Now, by a standard argument (see, for example, [11, pp. 317-319]), we can see that (3.42) holds for any $x_{1} \in$ $B^{+}\left(0, R_{2}\right) \cup \Gamma\left(R_{2}\right)$ and $r \in\left(0, r_{0}\right)$. Thus, by the Morrey's theorem on the Dirichlet growth, we can deduce the assertion.

\section{Convergence lemma and boundary regularity}

Lemma 4.1 (Convergence lemma with boundary value). Let $B^{+}:=B^{+}(1)$ and $\Gamma:=\Gamma(1)$. Let $A^{(v)}(x, u)=$ $A_{i j}^{(v) \alpha \beta}(x, u)$ be a sequence of continuous functions defined on $B^{+} \times \mathbb{R}^{n}$ converging uniformly to $A(x, u)=A_{i j}^{\alpha \beta}(x, u)$ and satisfying the following inequalities for positive constants $K, \lambda_{A}$ and a bounded continuous concave function $\omega_{A}$ with $\omega_{A}(0)=0$.

(A-1) $\left|A^{(v)}(x, u)\right| \leq K$,

(A-2) $A^{(v)} \xi \xi:=A_{i j}^{(v) \alpha \beta} \xi_{\alpha}^{i} \xi_{\beta}^{j} \geq \lambda_{A}|\xi|^{2}$ for all $(x, u, \xi) \in B^{+} \times \mathbb{R}^{n} \times \mathbb{R}^{m n}$,

(A-3) $\left|A^{(v)}(x, u)-A^{(v)}(y, v)\right| \leq \omega_{A}\left(|x-y|^{2}+|u-v|^{2}\right)$.

Let $p_{v}(x)$ be a sequence of continuous functions on $B^{+}$converging uniformly to a constant $p_{0} \geq 2$ which satisfies the following conditions.

(P-1) $p_{v}(x) \geq 2$,

$(\mathrm{P}-2)\left|p_{v}(x)-p_{v}(y)\right| \leq \omega_{1}(|x-y| / 2)=c_{p}|x-y|^{\sigma}$ for constants $c_{p}>0$ and $\sigma \in(0,1)$.

For some fixed $s>m$, let $\left\{h^{(v)}\right\}$ be a sequence in $W^{1, s}\left(B^{+}\right)$converging to $h$ in $W^{1, s}\left(B^{+}\right)$weakly. For each $v \in \mathbb{N}$, let $u^{(v)} \in W^{1, p_{v}(x)}$ be a local minimizer of

$$
\mathcal{F}^{(v)}\left(v ; B^{+}\right):=\int_{B^{+}}\left(A^{(v)}(x, v) D v D v\right)^{p_{v}(x) / 2} d x
$$

in the class

$$
\left\{w \in W^{1, p_{v}(x)}\left(B^{+}\right) ; w=h^{(v)} \text { on } \Gamma\right\} .
$$

Suppose that $u^{(v)} \rightarrow v$ in $L^{2}\left(B^{+}\right)$and that $\left\|u^{(v)}\right\|_{\infty} \leq M$ for some positive constant $M$. Then, $u^{v}$, or a subsequence that we also denote by the same symbol, is such that $u^{(v)} \rightarrow v$ in $W^{1,(1+\varepsilon) p_{0}}\left(B^{+}(R)\right)$ for some $\varepsilon>0$ and any $R \in$ $(0,1)$, and $v$ minimizes the functional

$$
\mathcal{F}_{0}\left(w ; B^{+}(R)\right):=\int_{B^{+}(R)}(A(x, w) D w D w)^{p_{0} / 2} d x
$$

in the class

$$
\left\{w \in W^{1, p_{0}}\left(B^{+}(R)\right) ; w=h \text { on } \Gamma(R)\right\} .
$$

Moreover, if $x_{v}$ is a singular point of $u^{(v)}$ and $x_{v} \rightarrow \bar{x}$, then $\bar{x}$ is a singular point of $v$.

Proof. We divide the proof into 3 parts.

Part 1 (Preliminary estimates and the convergence of $u(v)$ ). Since all assumptions are independent on the number $v$, all results in Section 2 are valid with common constants for all $u^{(v)}$. So, by Proposition 2.2 there exists a constant 
$\delta_{0}>0$ such that $\left|D u^{(v)}\right|^{\left(1+\delta_{0}\right) p_{v}(x)} \in L_{\text {loc }}^{1}\left(B^{+}\right)$, and by Corollary 2.5 we also have

$$
\int_{B^{+}(R)}\left|D u^{(v)}\right|^{\left(1+\delta_{0}\right) p_{v}(x)} d x \leq C_{3}(R)
$$

for some constant $C_{3}(R)$ which depends on $R$, but does not on $v$. Fixing such a constant $\delta_{0}>0$, let us choose $\delta_{2} \in\left(0, \delta_{0}\right)$ for which Corollary 2.3 holds. In what follows, let $\delta$ be a positive constant with $\delta<\delta_{2}$.

Since we are assuming that $p_{v}(x)$ converge uniformly to $p_{0}$ on $B^{+}$, we can assume without loss of generality that

(P-3) $p_{v}(x)$ satisfies that $2 \leq q_{1} \leq p_{v}(x) \leq q_{2}$ on $B^{+}$for some constants $q_{1}$ and $q_{2}$ with

$$
q_{1}(1+\delta) \geq q_{2}\left(1+\frac{\delta}{2}\right), \quad p_{0}\left(1+\frac{\delta}{2}\right) \geq q_{2} .
$$

By virtue of (4.1), (4.2) and the choice of $\delta$, we have

$$
\int_{B^{+}(R)}\left|D u^{(v)}\right|^{(1+\delta / 2) q_{2}} d x \leq C_{4}(R) .
$$

Since we are assuming that $\left\|u^{(v)}\right\|_{\infty} \leq M$, the estimate (4.3) implies that $u^{(v)}-\tilde{v}$ in $W^{1,(1+\delta / 2) q_{2}}\left(B^{+}(R)\right)$ for some $\tilde{v} \in W^{1,(1+\delta / 2) q_{2}}\left(B^{+}(R)\right)$ taking subsequence if necessary. On the other hand we are assuming that $u^{(v)} \rightarrow v$ in $L^{2}$, so we see that $v=\tilde{v}$ and that

$$
\begin{aligned}
& u^{(v)} \rightarrow v \quad \text { in } L^{(1+\delta / 2) q_{2}}\left(B^{+}(R)\right), \\
& D u^{(v)} \rightarrow D v \quad \text { in } L^{(1+\delta / 2) q_{2}}\left(B^{+}(R)\right) .
\end{aligned}
$$

Thus, we get the assertion on the convergence of $u^{(v)}$.

Moreover, by virtue of the lower semicontinuity of the norm with respect to weak convergence, we have

$$
\int_{B^{+}(R)}|D v|^{(1+\delta / 2) q_{2}} d x \leq C_{4}(R) .
$$

Part 2 (Minimality of $v$ ). Now, let us prove that $v$ minimizes $\mathcal{F}_{0}$ relative to the boundary value $h$ on $\Gamma(R)$. For this purpose, as the first step we are going to show that

$$
\mathcal{F}_{0}\left(v ; B^{+}(R)\right) \leq \liminf _{\nu \rightarrow \infty} \mathcal{F}^{(v)}\left(u^{(v)} ; B^{+}(R)\right) .
$$

Observing that

$$
\mathcal{F}^{(v)}\left(u^{(v)} ; B^{+}(R)\right)=\mathcal{F}_{0}\left(u^{(v)} ; B^{+}(R)\right)+\mathcal{F}^{(v)}\left(u^{(v)} ; B^{+}(R)\right)-\mathcal{F}_{0}\left(u^{(v)} ; B^{+}(R)\right),
$$

and mentioning the lower semicontinuity of $\mathcal{F}_{0}$ with respect to the weak convergence in $W^{1, p_{0}}\left(B^{+}(R)\right)$, we see that it is enough to show that

$$
\left|\mathcal{F}^{(v)}\left(u^{(v)} ; B^{+}(R)\right)-\mathcal{F}_{0}\left(u^{(v)} ; B^{+}(R)\right)\right| \rightarrow 0 \quad \text { as } v \rightarrow \infty .
$$

Let us put

$$
\begin{aligned}
& e_{v}:=A^{(v)}\left(x, u^{(v)}\right) D u^{(v)} D u^{(v)}, \\
& e_{1}:=A\left(x, u^{(v)}\right) D u^{(v)} D u^{(v)} .
\end{aligned}
$$

Then we have

$$
\begin{aligned}
& \left|\mathcal{F}^{(v)}\left(u^{(v)} ; B^{+}(R)\right)-\mathcal{F}_{0}\left(u^{(v)} ; B^{+}(R)\right)\right| \\
& \quad \leq \int_{B^{+}(R)}\left|e_{v}^{p_{v}(x) / 2}-e_{v}^{p_{0} / 2}\right| d x+\int_{B^{+}(R)}\left|e_{v}^{p_{0} / 2}-e_{1}^{p_{0} / 2}\right| d x \\
& =: I+I I .
\end{aligned}
$$


Put

$$
\tilde{p}_{v}(x):=\max \left\{p_{v}(x), p_{0}\right\} \quad\left(\leq q_{2}\right) .
$$

Then, by virtue of (2.22) and (4.3), taking $\varepsilon \leq \delta / 2$, we can see that

$$
\begin{aligned}
I & \leq c_{e}(\varepsilon) \int_{B^{+}(R)}\left|p_{v}(x)-p_{0}\right|\left(1+e_{v}\right)^{\tilde{p}_{v}(1+\varepsilon) / 2} d x \\
& \leq c_{e}(\varepsilon) \sup _{B^{+}(R)}\left|p_{v}(x)-p_{0}\right| \int_{B^{+}(R)}\left(1+e_{v}\right)^{q_{2}(1+\delta / 2) / 2} d x \\
& \leq c(\varepsilon, R) \sup _{B^{+}(R)}\left|p_{v}(x)-p_{0}\right| \rightarrow 0 \quad \text { as } v \rightarrow \infty .
\end{aligned}
$$

In order to estimate $I I$, we mention that for $q \geq 1$

$$
\left|s^{q}-t^{q}\right| \leq q|s-t|\left(s^{q-1}+t^{q-1}\right)
$$

holds for any $s, t \geq 0$. Then, using (4.3) also, and mentioning that $q_{2} \geq p_{0}$, we can estimate $I I$ as

$$
\begin{aligned}
I I & \leq c \int_{B^{+}(R)}\left|A^{(v)}\left(x, u^{(v)}\right)-A\left(x, u^{(v)}\right)\right| \cdot\left(1+\left|D u^{(v)}\right|\right)^{q_{2}} d x \\
& \leq c\left(\int_{B^{+}(R)}\left|A^{(v)}\left(x, u^{(v)}\right)-A\left(x, u^{(v)}\right)\right|^{(2+\delta) / \delta} d x\right)^{\delta /(2+\delta)}\left(\int_{B^{+}(R)}\left(1+\left|D u^{(v)}\right|\right)^{(1+\delta / 2) q_{2}} d x\right)^{2 /(2+\delta)} \\
& \leq c(R)\left(\int_{B^{+}(R)}\left|A^{(v)}\left(x, u^{(v)}\right)-A\left(x, u^{(v)}\right)\right|^{(2+\delta) / \delta} d x\right)^{\delta /(2+\delta)} .
\end{aligned}
$$

Since (4.4) implies $u^{(v)}(x) \rightarrow v(x)$ almost every $x$, taking subsequence if necessary, from the assumption that $A^{(v)}(x, u)$ converges uniformly to $A(x, u)$, by virtue of Lebesgue's dominated convergent theorem, we have that

$$
\int_{B^{+}(R)}\left|A^{(v)}\left(x, u^{(\nu)}\right)-A\left(x, u^{(\nu)}\right)\right|^{(2+\delta) / \delta} d x \rightarrow 0 .
$$

Thus we see that

$$
I I \rightarrow 0 \quad \text { as } v \rightarrow \infty .
$$

From (4.12), (4.14) and (4.16) we get (4.9), so we see that (4.7) holds.

Now, let us prove that $v$ is a local minimizer of $\mathcal{F}_{0}$. Let $w \in W^{1, p_{0}}\left(B^{+}(R)\right)$ be a minimizer of $\mathcal{F}_{0}$ on $B^{+}(R)$ with $w=v$ on $\partial B^{+}(R)$. We mention that the $w$ satisfies the same boundary condition that $v$ satisfies on $\Gamma(R)$, namely $w=h$ on $\Gamma(R)$.

In the following part of the proof, taking $v$ sufficiently large, we suppose always that

$$
\left(1+\frac{\delta}{2}\right) p_{0} \geq\left(1+\frac{\delta}{4}\right) \sup _{B^{+}} p_{v}(x) .
$$

On the other hand, by (4.4) and (4.5), we have that

$$
v \in W^{1,(1+\delta / 2) q_{2}}\left(B^{+}(R)\right) \subset W^{1,(1+\delta / 2) p_{0}}\left(B^{+}(R)\right) .
$$

Then, using Corollary 2.3 with $p(x)=p_{0}$, we see that

$$
w \in W^{1,(1+\delta / 2) p_{0}}\left(B^{+}(R)\right) \subset W^{1,(1+\delta / 4) p_{\nu}(x)}\left(B^{+}(R)\right) \cap W^{1, q_{2}}\left(B^{+}(R)\right) .
$$


Here, we used (4.2) and (4.17) for the last inclusion. Moreover, using (4.17), Corollary 2.3 and (4.6), we see that $w$ satisfies

$$
\int_{B^{+}(R)}|D w|^{(1+\delta / 4) p_{v}(x)} d x \leq c \int_{B^{+}(R)}(1+|D w|)^{(1+\delta / 2) p_{0}} d x \leq c \int_{B^{+}(R)}(1+|D v|)^{(1+\delta / 2) p_{0}} d x \leq c(R) .
$$

Fixing $R \in(0,1)$, for $\rho \in(R / 2, R)$ put

$$
T_{\rho}:=\left\{x \in B^{+}(\rho) ; x^{m}>R-\rho\right\},
$$

and let $\eta \in C_{0}^{1}\left(B^{+}(R)\right)$ be a cut-off function satisfying that

$$
0 \leq \eta \leq 1 \quad \text { on } B^{+}(R), \quad \eta \equiv 1 \quad \text { on } T_{\rho}, \quad|D \eta| \leq \frac{2}{R-\rho} \quad \text { on } B^{+}(R) .
$$

If necessary, we extend $\eta$ outside $B^{+}(R)$ by 0 . Let us put

$$
\psi:=(1-\eta)\left(u^{(v)}-v\right), \quad v^{(v)}:=w+\psi .
$$

From the assumption that $w=v$ on $\partial B^{+}(R)$, we have

$$
v^{(v)}=w+\left(u^{(v)}-v\right)=u^{(v)} \quad \text { on } \partial B^{+}(R) .
$$

So, the minimality of $u^{(v)}$ for $\mathcal{F}^{(v)}$, we see that

$$
\mathcal{F}^{(v)}\left(u^{(v)} ; B^{+}(R)\right) \leq \mathcal{F}^{(v)}\left(v^{(v)} ; B^{+}(R)\right) .
$$

Now, as in [6, pp. 458-460], by estimating $\left|\mathcal{F}^{(v)}\left(v^{(v)} ; B^{+}(R)\right)-\mathcal{F}_{0}\left(v^{(v)} ; B^{+}(R)\right)\right|$ and $\mid \mathcal{F}_{0}\left(v^{(v)} ; B^{+}(R)\right)-$ $\mathcal{F}_{0}\left(w ; B^{+}(R)\right) \mid$, we show that $\mathcal{F}^{(v)}\left(v^{(v)} ; B^{+}(R)\right) \rightarrow \mathcal{F}_{0}\left(w ; B^{+}(R)\right)$.

First, let us estimate $\left|\mathcal{F}^{(v)}\left(v^{(v)} ; B^{+}(R)\right)-\mathcal{F}_{0}\left(v^{(v)} ; B^{+}(R)\right)\right|$.

$$
\begin{aligned}
& \left|\mathcal{F}^{(v)}\left(v^{(v)} ; B^{+}(R)\right)-\mathcal{F}_{0}\left(v^{(v)} ; B^{+}(R)\right)\right| \\
& \leq \quad \int_{B^{+}(R)}\left|\left(A^{(v)}\left(x, v^{(v)}\right) D v^{(v)} D v^{(v)}\right)^{p_{v}(x) / 2}-\left(A\left(x, v^{(v)}\right) D v^{(v)} D v^{(v)}\right)^{p_{v}(x) / 2}\right| d x \\
& \quad+\int_{B^{+}(R)}\left|\left(A\left(x, v^{(v)}\right) D v^{(v)} D v^{(v)}\right)^{p_{v}(x) / 2}-\left(A\left(x, v^{(v)}\right) D v^{(v)} D v^{(v)}\right)^{p_{0} / 2}\right| d x \\
& \leq \quad \int_{B^{+}(R)}\left|A^{(v)}\left(x, v^{(v)}\right)-A(x, v(v))\right| \cdot\left|D v^{(v)}\right|^{p_{v}(x)} d x \\
& \quad+C(\varepsilon) \sup _{B^{+}(R)}\left|p_{v}(x)-p_{0}\right| \int_{B^{+}(R)}\left(1+\left|D v^{(v)}\right|^{2}\right)^{(1+\delta / 2) p_{0} / 2} d x,
\end{aligned}
$$

where we used (4.15) and (2.22). By the definition of $v^{(v)}$, we see that

$$
\left|D v^{(v)}\right|=\left|D w+D(1-\eta)\left(u^{(v)}-v\right)\right| \leq|D w|+(1-\eta)\left|D\left(u^{(v)}-v\right)\right|+\frac{2}{R-\rho}\left|u^{(v)}-v\right| .
$$

So,we have that

$$
\begin{aligned}
& \int_{B^{+}(R)}\left|D v^{(v)}\right|^{(1+\delta / 2) p_{0} / 2} d x \\
& \leq c\left(p_{0}\right)\left[\int_{B^{+}(R)}|D w|^{(1+\delta / 2) p_{0} / 2}+\int_{B^{+}(R)}\left|D u^{(v)}\right|^{(1+\delta / 2) p_{0} / 2} d x+\int_{B^{+}(R)}|D v|^{(1+\delta / 2) p_{0} / 2} d x\right. \\
& \left.\quad+\left(\frac{2}{R-\rho}\right)^{(1+\delta / 2) p_{0} / 2} \int_{B^{+}(R)}\left|u^{(v)}-v\right|^{(1+\delta / 2) p_{0} / 2} d x\right] .
\end{aligned}
$$


By virtue of (4.3), (4.4), (4.6) and (4.20), all terms of the right hand side can be estimated by some constant $C(R)$ depending on $R$. Thus we get

$$
\int_{B^{+}(R)}\left(1+\left|D v^{(v)}\right|^{2}\right)^{(1+\delta / 2) p_{0} / 2} d x \leq C(R) .
$$

By Hölder's inequality, (4.17) and (4.27) we can estimate the first term of the right-hand side of (4.24) as

$$
\begin{aligned}
& \int_{B^{+}(R)}\left|A^{(v)}\left(x, v^{(v)}\right)-A\left(x, v^{(v)}\right)\right| \cdot\left|D v^{(v)}\right|^{p_{v}(x)} d x \\
& \leq\left(\int_{B^{+}(R)}\left|A^{(v)}\left(x, v^{(v)}\right)-A\left(x, v^{(v)}\right)\right|^{(4+\delta) / \delta}\right)^{\delta /(4+\delta)}\left(\int_{B^{+}(R)}\left|D v^{(v)}\right|^{(1+\delta / 4) p_{v}} d x\right)^{4 /(4+\delta)} \\
& \leq\left(\int_{B^{+}(R)}\left|A^{(v)}\left(x, v^{(v)}\right)-A\left(x, v^{(v)}\right)\right|^{(4+\delta) / \delta}\right)^{\delta /(4+\delta)}\left(\int_{B^{+}(R)}\left(1+\left|D v^{(v)}\right|^{2}\right)^{(1+\delta / 2) p_{0} / 2} d x\right)^{4 /(4+\delta)} \\
& \leq C(R)\left(\int_{B^{+}(R)}\left|A^{(v)}\left(x, v^{(v)}\right)-A\left(x, v^{(v)}\right)\right|^{(4+\delta) / \delta} d x\right)^{\delta /(4+\delta)} .
\end{aligned}
$$

By (4.4) and the assumption that $A^{(v)}$ converges uniformly to $A$, we see that the right-hand side of (4.28) tends to 0 as $v \rightarrow \infty$. From (4.27), we also see that the second term of (4.24) tends to 0 as $v \rightarrow \infty$ easily. Thus we have

$$
\lim _{\nu \rightarrow \infty}\left|\mathcal{F}^{(v)}\left(v^{(\nu)} ; B^{+}(R)\right)-\mathcal{F}_{0}\left(v^{(\nu)} ; B^{+}(R)\right)\right|=0 .
$$

Next, let us estimate $\left|\mathcal{F}_{0}\left(v^{(v)}\right) ; B^{+}(R)-\mathcal{F}_{0}\left(w ; B^{+}(R)\right)\right|$. Remarking that $w$ differs from $v^{(v)}$ only on $B^{+}(R) \backslash T_{\rho}$, and using (4.25), we see that

$$
\begin{aligned}
\left|\mathcal{F}_{0}\left(v^{(v)} ; B^{+}(R)\right)-\mathcal{F}_{0}\left(w ; B^{+}(R)\right)\right| & \left|\int_{B^{+}(R)}\left(A\left(x, v^{(v)}\right) D v^{(v)} D v^{(v)}\right)^{p_{0} / 2} d x-\int_{B^{+}(R)}(A(x, w) D w D w)^{p_{0} / 2} d x\right| \\
\leq & K \int_{B^{+}(R) \backslash T_{\rho}}\left|D v^{(v)}\right|^{p_{0}} d x+K \int_{B^{+}(R) \backslash T_{\rho}}|D w|^{p_{0}} d x \\
\leq & 2 K C\left(p_{0}\right)\left[\int_{B^{+}(R) \backslash T_{\rho}}|D w|^{p_{0}} d x+\int_{B^{+}(R) \backslash T_{\rho}}\left|D u^{(v)}\right|^{p_{0}} d x\right. \\
& \left.+\int_{B^{+}(R) \backslash T_{\rho}}|D v|^{p_{0}} d x+\left(\frac{2}{R-\rho}\right)^{p_{0}} \int_{B^{+}(R) \backslash T_{\rho}}\left|u^{(v)}-v\right|^{p_{0}} d x\right] \\
= & : I I I+I V+V+V I
\end{aligned}
$$

where $K$ is a constant which appeared in condition (A-1) and $c\left(p_{0}\right)$ a constant depending only on $p_{0}$. Since the weak convergence (4.5) implies uniform boundedness of $L^{(1+\delta / 2) q_{2}}$ norm, we see that there exists a constant $M_{0}$ such that

$$
\left(\int_{B^{+}(R)}\left|D u^{(v)}\right|^{(1+\delta / 2) p_{0}} d x\right)^{2 /(2+\delta)} \leq M_{0} .
$$

Here, mention that $p_{0} \leq q_{2}$. So, using Hölder's inequality, we can estimate $I V$ as 


$$
\begin{aligned}
I V & \leq\left(\int_{B^{+}(R) \backslash T_{\rho}} 1 d x\right)^{\delta /(2+\delta)}\left(\int_{B^{+}(R) \backslash T_{\rho}}\left|D u^{(v)}\right|^{(1+\delta / 2) p_{0}} d x\right)^{2 /(2+\delta)} \\
& \leq c\left(R^{m}-\rho^{m}\right)^{\delta /(2+\delta)} M_{0} .
\end{aligned}
$$

Similarly, by virtue of (4.18) and (4.19), using Hölder's inequality, we can estimate $I I I$ and $V$ as follows

$$
\begin{aligned}
& I I I \leq C\left(R^{m}-\rho^{m}\right)^{\delta /(2+\delta)}\|D w\|_{L^{(1+\delta / 2) p_{0}\left(B^{+}(R)\right)},}^{p_{0}}, \\
& V \leq C\left(R^{m}-\rho^{m}\right)^{\delta /(2+\delta)}\|D v\|_{L^{(1+\delta / 2) p_{0}\left(B^{+}(R)\right)}}^{p^{(}{ }^{2}} .
\end{aligned}
$$

For fixed $R$ and $\rho$, the strong convergence (4.4) implies that

$$
V I \rightarrow 0 \quad \text { as } v \rightarrow \infty
$$

Combing (4.30) with (4.32)-(4.35), we see that

$$
\limsup _{v \rightarrow \infty}\left|\mathcal{F}_{0}\left(v^{(v)} ; B^{+}(R)\right)-\mathcal{F}_{0}(w)\right| \leq M_{1}\left(M_{0}, v, w\right)\left(R^{m}-\rho^{m}\right)^{\delta /(2+\delta)} .
$$

Now, by virtue of (4.7), (4.23), (4.29) and (4.36), we obtain

$$
\begin{aligned}
\mathcal{F}_{0}\left(v ; B^{+}(R)\right) & \leq \liminf _{\nu \rightarrow \infty} \mathcal{F}^{(v)}\left(u^{(v)}, B^{+}(R)\right) \\
& \leq \liminf _{\nu \rightarrow \infty} \mathcal{F}^{(v)}\left(v^{(v)}, B^{+}(R)\right) \\
& =\liminf _{\nu \rightarrow \infty} \mathcal{F}_{0}\left(v^{(v)}, B^{+}(R)\right) \\
& \leq \mathcal{F}_{0}\left(w ; B^{+}(R)\right)+C M_{1}\left(R^{m}-\rho^{m}\right)^{\delta /(2+\delta)}
\end{aligned}
$$

Letting $\rho \rightarrow R$, we see that $\mathcal{F}_{0}\left(v ; B^{+}(R)\right) \leq \mathcal{F}_{0}\left(w ; B^{+}(R)\right)$. On the other hand we are assuming that $w$ minimizes $\mathcal{F}_{0}$ relative to the boundary value $w=v$ on $\partial B^{+}(R)$. So, we can conclude that $v$ minimizes $\mathcal{F}_{0}$.

Part 3 (Proof for the statement on singular points). Let $x_{v} \in B^{+} \cup \Gamma$ be a singular point of $u^{(v)}$ and assume that $x^{\nu} \rightarrow \bar{x}$. We want to show that $\bar{x}$ is a singular point of the limit map $v$. For the case that $\bar{x} \in B^{+}$this assertion is shown in [16]. So let us consider the case $\bar{x} \in \Gamma_{R}$ for some $R \in(0,1)$.

Considering sufficiently large $v$ if necessary, we can assume that $x^{\nu} \in B^{+}\left(R^{\prime}\right)$ for some $R^{\prime} \in(R, 1)$.

For $y \in B^{+}\left(R^{\prime \prime}\right)$ and $r \in\left(0,1-R^{\prime \prime}\right)$, let us write

$$
p_{2}^{(\nu)}(y, r):=\sup _{\Omega(y, r)} p_{v}(x) .
$$

By virtue of Theorem 3.2, we can choose $\bar{R} \in\left(0,1-R^{\prime \prime}\right)$ so that

$$
\Psi_{v}\left(x_{v}, r\right):=r^{\rho_{2}^{(v)}\left(x_{v}, r\right)-m} \int_{\Omega\left(x_{v}, r\right)}\left(1+|D u|^{2}\right)^{p_{2}^{(v)}\left(x_{v}, r\right) / 2} d x>\varepsilon_{0}
$$

holds for the positive number $\varepsilon_{0}$ that appears in Theorem 3.2, any $r \in(0, \bar{R})$ and any number $v \in \mathbb{N}$. In the following, let us abbreviate

$$
p_{2}^{(v)}(r):=p_{2}^{(v)}\left(x_{v}, r\right)=\sup _{\Omega\left(x_{v}, r\right)} p_{v}(x),
$$

and let $\delta^{\prime}<\delta$ be a positive constant satisfying

$$
\delta^{\prime} q_{2} \leq \frac{\sigma}{2}
$$

Since $p_{v}(x) \rightarrow p_{0}$ uniformly, taking $v$ sufficiently large, we can assume that

$$
\left(1+\frac{\delta^{\prime}}{2}\right) p_{2}^{(v)}(r) \leq\left(1+\delta^{\prime}\right) p_{v}(x) \quad \text { for all } x \in \Omega\left(x_{v}, r\right) \subset B .
$$


Using (2.22) and Corollary 2.5, we see that

$$
\begin{aligned}
& r_{2}^{p_{2}^{(v)}(r)-m}\left|\int_{\Omega\left(x_{v}, r\right)}\left(1+\left|D u^{(v)}\right|^{2}\right)^{p_{2}^{(\nu)}(r) / 2} d x-\int_{\Omega\left(x_{v}, r\right)}\left(1+\left|D u^{(v)}\right|^{2}\right)^{p_{\nu}(x) / 2} d x\right| \\
& \leq r^{p_{2}^{(\nu)}(r)-m} \omega_{1}(r) c_{e}\left(\delta^{\prime} / 2\right) \int_{\Omega\left(x_{v}, r\right)}\left(1+\left|D u^{(v)}\right|^{2}\right)^{\left(1+\delta^{\prime} / 2\right) p_{2}^{(v)}(r) / 2} d x \\
& \leq r^{p_{2}^{(\nu)}(r)} \omega_{1}(r) c_{e}\left(\delta^{\prime} / 2\right) f_{\Omega\left(x_{v}, r\right)}\left(1+\left|D u^{(v)}\right|^{2}\right)^{\left(1+\delta^{\prime}\right) p_{v}(x) / 2} d x \\
& \leq c r^{\sigma} r_{2}^{p_{2}^{(v)}(r)} r^{-\left(1+\delta^{\prime}\right) p_{2}^{(v)}(4 r)} \leq c r^{\sigma-\left(1+\delta^{\prime}\right) \omega_{1}(4 r)-\delta^{\prime} q_{2}} \\
& \leq c r^{\sigma / 2-\left(1+\delta^{\prime}\right) \omega_{1}(4 r)} \rightarrow 0 \text { as } r \rightarrow 0,
\end{aligned}
$$

since $\omega_{1}(4 r) \rightarrow 0$ as $r \rightarrow 0$. Here, we used (4.41) for the second inequality, (2.12) and assumption (P-2) for the third one and (4.40) for the last one. Thus, (4.39) implies

$$
r^{p_{2}^{(v)}\left(x_{v}, r\right)-m} \int_{\Omega\left(x_{\nu}, r\right)}\left(1+\left|D u^{(\nu)}\right|^{2}\right)^{p_{\nu}(x) / 2} d x \geq \varepsilon_{0} / 2, \quad \text { for any } r \in(0, \tilde{R}),
$$

for sufficiently small $\tilde{R} \in(0, \bar{R})$.

Remarking that

$$
(a+b)^{q} \leq 2^{q-1}\left(a^{q}+b^{q}\right), \quad \sqrt{a+b} \leq \sqrt{a}+\sqrt{b} \text { for any } a, b \geq 0 \text { and } q \geq 1,
$$

and taking $r>$ so small that

$$
2^{q_{2}-1} r_{2}^{p_{2}^{(v)}(r)} \omega_{m}<\frac{\varepsilon_{0}}{8}
$$

we get

$$
r^{p_{2}^{(v)}\left(x_{v}, r\right)-m} \int_{\Omega\left(x_{v}, r\right)}\left|D u^{(v)}\right|^{p_{v}(x)} d x \geq \frac{3}{8 \cdot 2^{q_{2}-1}} \varepsilon_{0}=\frac{3}{2^{q_{2}+2}} \varepsilon_{0} .
$$

Here, $\omega_{m}$ stands for the volume of $m$-dimensional unit ball.

Thus, for singular points $x_{v}$ of $u^{(v)}$, combining (4.43) with (2.9), we see that

$$
c_{4} r^{p_{2}^{(\nu)}(r)-m}\left(\int_{\Omega\left(x_{v}, 2 r\right)}\left|\frac{u^{(\nu)}-h^{(\nu)}}{2 r}\right|^{p_{\nu}(x)} d x+\int_{\Omega\left(x_{v}, 2 r\right)}\left|D h^{(v)}\right|^{p_{\nu}(x)}\right) \geq \frac{3}{2^{q_{2}+2}} \varepsilon_{0} .
$$

Since we are assuming that $h^{(v)} \in W^{1, s}$ for some $s>m$, we have

$$
\begin{aligned}
& \int_{\Omega\left(x_{v}, 2 r\right)}\left|D h^{(v)}\right|^{p_{v}(x)} \\
& \leq \int_{\Omega\left(x_{\nu}, 2 r\right)}\left(1+\left|D h^{(v)}\right|\right)^{p_{2}^{(\nu)}(2 r)} d x \\
& \leq 2^{p_{2}^{(\nu)}(2 r)}\left[(2 r)^{m} \omega_{m}+\left[(2 r)^{m} \omega_{m}\right]^{1-p_{2}^{(\nu)}(2 r) / s}\left(\int_{\Omega\left(x_{v}, 2 r\right)}\left|D h^{(v)}\right|^{s} d x\right)^{p_{2}^{(\nu)}(2 r) / s}\right] .
\end{aligned}
$$

On the other hand we are also assuming that $h^{(v)}$ converges weakly to $h$ in $W^{1, s}\left(B^{+}\right)$, so $\int_{\Omega\left(x_{v}, 2 r\right)}\left|D h^{(v)}\right|^{s} d x$ are bounded by a constant which does not depend on $v$ and $r$. Thus, remarking also that $p_{2}^{(v)} \geq q_{1}$ and that $r<1$ we have 
for a constant $C_{h}$ that

$$
r^{p_{2}^{(v)}(2 r)-m} \int_{\Omega\left(x_{v}, 2 r\right)}\left|D h^{(v)}\right|^{p_{v}(x)} d x \leq C_{h} r^{q_{1}(1-m / s)} .
$$

Now, choosing $r>0$ sufficiently small so that

$$
c_{4} C_{h} r^{q_{1}(1-m / s)} \leq \frac{1}{2^{q_{2}+2}} \varepsilon_{0},
$$

we obtain from (4.44) that

$$
r^{p_{2}^{(v)}(r)-m} \int_{\Omega\left(x_{v}, 2 r\right)}\left|\frac{u^{(\nu)}-h^{(v)}}{2 r}\right|^{p_{v}(x)} d x \geq \frac{1}{2^{q_{2}+2} c_{4}} \varepsilon_{0} .
$$

On the other hand, since $x_{v} \rightarrow \bar{x}, h^{(v)} \rightarrow h$ in $L^{q_{2}}, p_{v}(x) \rightrightarrows p_{0}$ and $u^{(v)} \rightarrow v$ in $L^{q_{2}}$, we can see that, as in [16, (3.41)],

$$
r^{-m} \int_{\Omega\left(x_{\nu}, r\right)}\left|u^{(v)}-h^{(v)}\right|^{p_{v}(x)} d x \rightarrow r^{-m} \int_{\Omega(\bar{x}, R)}|v-h|^{p_{0}} d x .
$$

So, from (4.45) we can deduce that

$$
r^{-m} \int_{\Omega(\bar{x}, r)}|v-h|^{p_{0}} d x \geq \frac{1}{2^{q_{2}+2} c_{4}} \varepsilon_{0}>0
$$

for any $r \in(0, \tilde{R})$ for some $\tilde{R}>0$. This implies that $\bar{x}$ is a singular point of $v$, since $v=h$ on the boundary.

Now, thanks to the above lemma, we can prove full boundary regularity, Theorem 1.1, proceeding as in [6].

Proof of Theorem 1.1. For an arbitrarily fixed point $x_{0} \in \partial \Omega$, choose a positive number $R_{1}>0$ sufficiently small so that (3.6) in Theorem 3.2 holds. By considering suitable bi-Lipschitz transformation from $B\left(x_{0}, R_{1}\right)$ onto $B^{+}=$ $B^{+}(0,1)$, we can assume, without loss in generality, that $x_{0}=0, B^{+}=B\left(x_{0}, R_{1}\right) \cap \Omega$ and that (3.6) holds on $B^{+}$. It is enough to show that $x_{0}=0$ is not a singular point of $u$.

For $v \in \mathbb{N}$, let us put

$$
\begin{aligned}
& u^{(v)}(x):=u\left(v^{-1} x\right), \quad h^{(v)}(x):=h\left(v^{-1} x\right), \quad p_{v}(x):=p\left(v^{-1} x\right) \\
& A^{(v)}(x, v)=A_{i j}^{(v) \alpha \beta}(x, v):=v^{2-2\left(p(0) / p_{v}(x)\right)} g^{\alpha \beta}\left(v^{-1} x\right) h_{i j}(v) .
\end{aligned}
$$

Then, $u^{(v)}$ minimizes the functional

$$
\mathcal{E}^{(v)}\left(v ; B^{+}\right):=\int_{B^{+}}\left(A_{i j}^{(v) \alpha \beta}(x, v) D_{\alpha} v^{i} D_{\beta} v^{j}\right)^{p_{(v)}(x) / 2} d x,
$$

in the class

$$
\left\{v \in W^{1, p_{v}(x)}\left(B^{+}\right) ; v=h \text { on } \Gamma\right\} .
$$

Since we are assuming that $p(x)$ is Hölder continuous, $v^{p_{(v)}(x)-p(0)}$ tends to 1 uniformly as $v \rightarrow \infty$. So, we have that

$$
A_{i j}^{(v) \alpha \beta}(x, v) \rightrightarrows g^{\alpha \beta}(0) h_{i j}(v) .
$$

On the other hand, since we are assuming the boundedness of $u,\left\|u^{(v)}\right\|_{\infty}$ are uniformly bounded, and therefore, taking subsequence if necessary, $u^{(v)} \rightarrow u_{\infty}$ for some $u_{\infty}$ in $L^{2}\left(B^{+}\right)$.

About the boundary conditions $h^{(v)}$, we can see that $h^{(v)} \rightarrow h(0)$ strongly in $W^{1, s}\left(B^{+}\right)$exactly as in [6, p. 465]. 
Thus, all the assumptions in Lemma 4.1 are satisfied. So, using Lemma 4.1, we see that $u_{\infty}$ minimizes the functional

$$
\mathcal{E}_{\infty}\left(v, B^{+}\right):=\int_{B^{+}}\left(g^{\alpha \beta}(0) h_{i j}(v) D_{\alpha} v^{i} D_{\beta} v^{j}\right)^{p(0) / 2} d x,
$$

in the class

$$
\left\{v \in W^{1, p(0)}\left(B^{+}\right) ; v=h(0) \text { on } \Gamma\right\},
$$

and 0 is a singular point of $u_{\infty}$. However, [6, Theorem 5.4] says that a minimizer of a standard $p$-growth functional $(p>1)$ cannot have singularity on the boundary. This is a contradiction, and we conclude that $x_{0}=0$ cannot be a singular point of $u$.

\section{Conflict of interest statement}

This article has no conflict of interest.

\section{Acknowledgement}

This work was partly supported by JSPS Grant-in-Aid for Scientific Research (C) "KAKENHI” Grant Number 26400177.

\section{References}

[1] E. Acerbi, G. Mingione, Regularity results for a class of functionals with non-standard growth, Arch. Ration. Mech. Anal. 156 (2) (2001) 121-140.

[2] E. Acerbi, G. Mingione, Regularity results for a class of quasiconvex functionals with nonstandard growth, Ann. Sc. Norm. Super. Pisa Cl. Sci. (4) 30 (2) (2001) 311-339.

[3] E. Acerbi, G. Mingione, Regularity results for stationary electro-rheological fluids, Arch. Ration. Mech. Anal. 164 (3) (2002) $213-259$.

[4] A. Coscia, G. Mingione, Hölder continuity of the gradient of $p(x)$-harmonic mappings, C. R. Acad. Sci. Paris Sér. I Math. 328 (4) (1999) 363-368.

[5] A. Coscia, D. Mucci, Integral representation and $\Gamma$-convergence of variational integrals with $P(X)$-growth, ESAIM Control Optim. Calc. Var. 7 (2002) 495-519 (electronic).

[6] F. Duzaar, J.F. Grotowski, M. Kronz, Partial and full boundary regularity for minimizers of functionals with nonquadratic growth, J. Convex Anal. 11 (2) (2004) 437-476.

[7] M. Eleuteri, Hölder continuity results for a class of functionals with non-standard growth, Boll. Unione Mat. Ital., Sez. B, Artic. Ric. Mat. (8) 7 (1) (2004) 129-157.

[8] M. Eleuteri, J. Habermann, Calderón-Zygmund type estimates for a class of obstacle problems with $p(x)$ growth, J. Math. Anal. Appl. 372 (1) (2010) 140-161.

[9] M. Eleuteri, J. Habermann, A Hölder continuity result for a class of obstacle problems under non standard growth conditions, Math. Nachr. $284(11-12)$ (2011) 1404-1434.

[10] M. Giaquinta, L. Martinazzi, An Introduction to the Regularity Theory for Elliptic Systems, Harmonic Maps and Minimal Graphs, second edition, Lecture Notes. Scuola Normale Superiore di Pisa (New Series), vol. 11, Edizioni della Normale, Pisa, 2012.

[11] E. Giusti, Direct Methods in the Calculus of Variations, World Scientific Publishing Co. Inc., River Edge, NJ, 2003.

[12] J. Jost, M. Meier, Boundary regularity for minima of certain quadratic functionals, Math. Ann. 262 (4) (1983) 549-561.

[13] P. Marcellini, Regularity of minimizers of integrals of the calculus of variations with nonstandard growth conditions, Arch. Ration. Mech. Anal. 105 (3) (1989) 267-284.

[14] M.A. Ragusa, A. Tachikawa, On interior regularity of minimizers of $p(x)$-energy functionals, Nonlinear Anal. 93 (2013) $162-167$.

[15] M.A. Ragusa, A. Tachikawa, H. Takabayashi, Partial regularity of $p(x)$-harmonic maps, Trans. Am. Math. Soc. 365 (6) (2013) $3329-3353$.

[16] A. Tachikawa, On the singular set of minimizers of $p(x)$-energies, Calc. Var. Partial Differ. Equ. 50 (1-2) (2014) 145-169.

[17] V.V. Zhikov, On Lavrentiev's phenomenon, Russ. J. Math. Phys. 3 (2) (1995) 249-269.

[18] V.V. Zhikov, On some variational problems, Russ. J. Math. Phys. 5 (1997) 105-116. 